\title{
An approach to service provisioning with quality of service requirements in ATM networks
}

\author{
Panagiotis Thomas* \\ Department of Electrical Engineering and Computer Science, University of Michigan, Ann Arbor, \\ MI 48109-2122, USA \\ Tel.: + 1313764 7568; E-mail: grthomas@umich.edu \\ Demosthenis Teneketzis \\ Department of Electrical Engineering and Computer Science, University of Michigan, Ann Arbor, \\ MI 48109-2122, USA \\ E-mail: teneket@umich.edu
}

\begin{abstract}
We present an approach to Service Provisioning with Quality of Service (QoS) requirements in ATM networks. The ATM network provides services to users every $\widehat{T}$ units of time. Each service is characterized by its one way route, its input traffic constraints and its provided QoS. The input traffic constraints consist of the maximum input rate and an upper bound on the burstiness curve. The QoS provided is defined by the maximum percentage of cell loss along the route and the maximum end-to-end cell delay. The service provisioning problem is defined as follows: determine the amount, price and required resources (bandwidth and buffers) for each type of service, that maximize a social welfare function that consists of the network's revenue and the users' surplus. This problem is solved every $\widehat{T}$ units of time and the allocation is made only over the available resources, that is, those that are not used by connections that are still active. We prove the existence of a solution to the Service Provisioning Problem and we also suggest an iterative procedure that interprets the solution. The network adjusts the prices to maximize the welfare function and also to guarantee that the allocated resources do not exceed the available resources. Based on these prices, users request a new allocation that minimizes their cost and the network adjusts again the prices based on the new allocation. The above procedure has the following features: (i) the network needs to know only the average request rate for each type of service, their route and the resources requested by the users; (ii) users need to know only their private information (input traffic constraints and QoS requirements), their route, and the prices for resources announced by the network.
\end{abstract}

\section{Introduction}

Asynchronous transfer mode (ATM) technology is widely regarded as the switching and multiplexing technique for broadband integrated services digital networks (B-ISDN). The development of these networks is driven by the emerging needs to provide real-time transport capabilities necessary for future multimedia applications incorporating voice, video and high speed data.

In this study we are concerned with the problem of allocating resources (buffers and bandwidths) in ATM networks so that the services provided by the network meet certain quality requirements expressed by maximum percentage of cell loss and maximum end-to-end cell delay. Resource reservation has been proposed to be the preferred approach to guarantee quality of service (QoS) in high speed applications. Most of the proposed

\footnotetext{
${ }^{*}$ Corresponding author.
} 
resource reservation schemes and their analysis so far are restricted to a single node (see, for example, [3-6, $8-13,21,25,27])$. Exceptions are $[2,7,14,20,22]$, which analyze resource reservation schemes along fixed paths consisting of multiple nodes. The work reported in $[2,7,14,22]$ is based on deterministic models whereas the study in [20] is based on a stochastic model.

Our approach to developing a methodology to service provisioning with QoS requirements in ATM networks builds on the method proposed by Low and Varaiya $[15,16,18]$. The salient features of Low's and Varaiya's approach are: (1) the ATM network offers several types of services differentiated by user traffic characteristics and quality parameters; (2) the network offers for rent bandwidth and buffers and users purchase these resources to meet their desired quality; (3) the objective is to maximize a welfare function subject to constraints that are dictated by: (i) the available resources; (ii) the requirement that no cell loss is allowed during the data transfer; and (iii) the maximum end-to-end delay (see [16]).

Our major contribution in this paper is the incorporation, in the resource allocation process, of the QoS requirements expressed by the maximum percentage of cell loss $L_{s}$ and the maximum end-to-end cell delay $D_{s}$ for each type of service $s$. The incorporation of the QoS requirements in the service provisioning process is achieved by taking a worst case approach to the percentage of cell loss and the end-to-end cell delay. This approach reveals the interaction among resource allocation, percentage of cell loss and end-to-end cell delay. Service provisioning is achieved by a mechanism similar to the one described in [16]; the network offers for rent its resources (bandwidth and buffers) and the users purchase them to meet their QoS requirements described above.

Our analysis of quality of service can also be used to address the admission control problem in ATM networks as follows: When the network receives a request for the admission of a certain type of service, it inspects its available resources (bandwidth and buffers) and attempts to establish a route (connection) from the source to the destination of the service request, so that there are sufficient available resources along that route to satisfy the quality of service requirements on percentage of cell losses and end-to-end cell delay. If the network can identify such a route it accepts the service request, otherwise it rejects it.

The remainder of the paper is organized as follows: in Section 2 we formulate the service provisioning with QoS requirements problem as a mathematical programming problem that has to be solved every $\widehat{T}$ units of time. In Section 3 we establish explicit expressions of the constraints on maximum percentage of cell loss and maximum end-to-end cell delay, needed to guarantee that the offered services will satisfy their QoS requirements. In Section 4 we present a solution procedure for the optimization problem of Section 2. In Section 5 we summarize the results, delineate their practical implications and discuss some open problems arising from our approach.

\section{Problem formulation}

We consider the network model of Fig. 1. It consists of a set $L$ of links. Link $l \in L$ comprises a transmission capacity of $C_{l}$ cps and a buffer of $B_{l}$ cells. $B_{l}$ is the size of the buffer at the preceding node that is supplied for link $l$. Therefore output buffering is assumed at each node. Both bandwidth and buffer are supplied for the direction of transmission shown in Fig. 1.

For our purposes, an ATM network provides a one-way connection between two end-points over a route for a certain duration of time. A route between its end-points spans several nodes and links. A connection is used to transport a data stream or message from one end-point to the other. The bandwidth and buffers, allocated to a connection can vary over the links in its route. Whenever the message rate at a node exceeds the allocated bandwidth, the excess cells are buffered and if the buffer is full they are lost.

When a service request is made, the network must decide (1) whether to admit that request; and if it is admitted, the network (2) must assign a connection to it, and (3) provide enough bandwidth and buffer resources to meet service quality. Decision (1) is usually called admission control, (2) is called routing, and (3) is called resource allocation or service provisioning. 


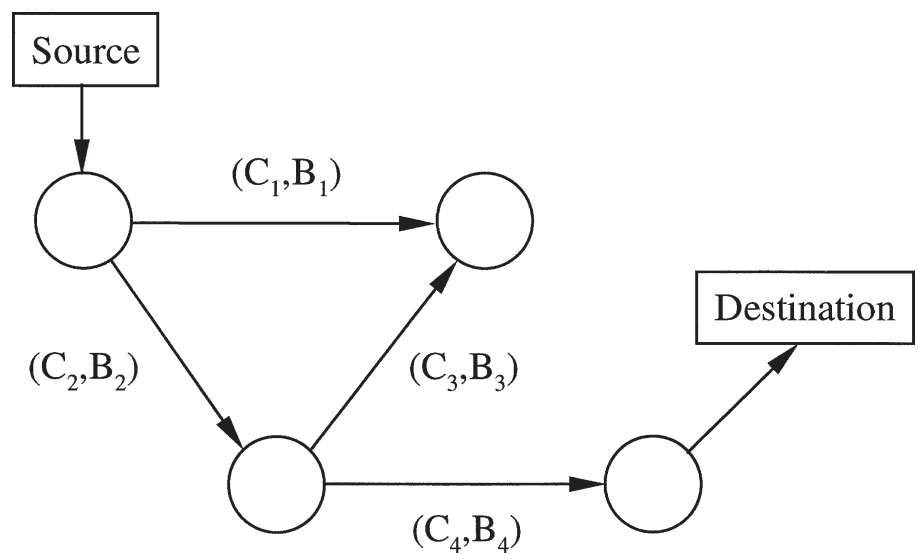

Fig. 1. Network model.

We make the following assumptions:

(A1) Time is divided into intervals of length $\widehat{T}$. Allocation decisions are made at the time instants $k \widehat{T}$, $k=0,1,2, \ldots$

(A2) At the time instants $k \widehat{T}, k=0,1,2, \ldots$, where allocation decisions are made, resources are not reserved in anticipation of future requests.

(A3) Incoming traffic to the network (i.e., input messages to the network) is modeled as a deterministic 'fluid flow' $m(\cdot)$ which represents the instantaneous rate in cps. Therefore $m(\cdot)$ is a nonnegative bounded function:

$$
0 \leqslant m(t) \leqslant \mu_{0}, \quad \forall t \in[0, T],
$$

where $\mu_{0} \triangleq \sup _{0 \leqslant t \leqslant T} m(t)$, and $T$ is the duration of the message.

(A4) Input messages are characterized by the Message Burstiness Function which is given by the following formula:

$$
b_{m}(\mu) \triangleq \sup _{0 \leqslant s \leqslant t \leqslant T} \int_{s}^{t}[m(\tau)-\mu] \mathrm{d} \tau, \quad \mu \geqslant 0 .
$$

(A5) The network offers a set $S$ of different types of services. Each type $s \in S$ of service is characterized by the maximum cell rate $\mu_{0}^{s}$, the maximum burstiness curve $b_{s}(\cdot)$, and its route. A type $s$ message that is a message that requests a service of type $s$ - must satisfy the following traffic constraints:

$$
\begin{array}{ll}
m(t) \leqslant \mu_{0}^{s}, & \forall t \in[0, T], \\
b_{m}(\mu) \leqslant b_{s}(\mu), & \forall \mu \in\left[0, \mu_{0}^{s}\right] .
\end{array}
$$

(A6) A type $s \in S$ service is sold by the network at a price $w_{s}$ per unit and is provided by a connection over a fixed route $r_{s}$, that is a set of links $l_{s} \in L$, throughout its duration.

(A7) The quality of service supplied to a type $s$ service is characterized by the following parameters, that are defined over the allocation period $\widehat{T}$ :

(i) the maximum percentage of cell loss $L_{s}$,

(ii) the maximum end-to-end cell delay $D_{s}$ (in units of time).

(A8) The demand (or user requests) for a type $s$ service is given by the aggregate request function $R_{s}\left(w_{s}\right)=$ $\lambda_{s} \widehat{T} \exp \left(-w_{s}\right)$, where $\lambda_{s} \exp \left(-w_{s}\right)$ denotes the arrival rate of a type $s$ request when the price per unit of type $s$ service is $w_{s}$. The nonnegative number $\lambda_{s}$ is known by the network. 
(A9) Users inform the network at the end of their service. The resources allocated to these users are then released to the network, that uses them on the next service allocation decision.

(A10) At the time instants $k \widehat{T}, k=0,1,2, \ldots$, where allocation decisions are made, the network reallocates only those resources that are freed by the termination of services in $((k-1) \widehat{T}, k \widehat{T}]$.

(A11) There is no sharing of the allocated resources (bandwidth and buffers) at each ATM switch, among the different connections that pass through the switch.

We now discuss and critique each of the above assumptions. The selection of the length $\widehat{T}$ of each allocation period must take into account the overall delay constraints (end-to-end delay plus delay due to waiting for the next allocation period) of the different types of services, the arrival rates of incoming traffic as well as the duration of the different types of services. The determination of the optimal (or even near optimal) value of $\widehat{T}$ is a challenging problem that will not be addressed in this paper. Here we assume that the value of $\widehat{T}$ is fixed and given to us. Neglecting future requests at allocation instances usually leads to suboptimal allocations. On the other hand, anticipation of future requests at allocation instances requires certain information, such as probability distribution functions on the duration of different types of services or probability distribution functions of the incoming traffic, which may not in general be available to us. Assumptions (A3)-(A5) provide a mathematical description of the incoming traffic. The small, fixed-sized packets that are used to carry the information in ATM networks justify the traffic approximation described by (A3). The description of input messages by their burstiness function (A4) is by now accepted as a practically reasonable approach to characterizing input traffic. The message burstiness $b_{m}(\mu)$ is interpreted as the minimum buffer size of a fixed rate $-\mu$ cps - server that is sufficient to prevent cell loss during the transmission of that message. The function $b_{m}(\cdot)$ is nonnegative, convex, strictly decreasing for $\mu<\mu_{0}$, and $b_{m}(\mu)=0$ for $\mu \geqslant \mu_{0}$ (see [15]). The definition of message burstiness is implicitly related to the tradeoff between bandwidth and buffers as substitutable resources for a specific service quality on a single node. We can achieve the same quality requirements by increasing the bandwidth and decreasing the buffers or vice versa. An assumption more realistic than (A5) would not distinguish among services that have the same characteristics (maximum rate, maximum burstiness) and the same quality of service requirements (cf. (A7)) but have different source and destination and different routes. Assumption (A6) implies that the routing of messages has already been decided and allows us to concentrate on the resource allocation (service provisioning) problem. Assumption (A7) explicitly describes the quality of service requirements on each type of service. In the literature, [25], jitter is also included in the quality parameters used to characterize the supplied quality of a type $s$ service. In our situation it will become evident that jitter is zero as a result of our problem formulation. In (A8) we have selected a family of strictly decreasing, convex functions to represent the users' demand for the different types of services. The following assumptions are implied by the choice of these functions: (i) each user can only request one type of service; (ii) there is no interdependence between the demands for different services, i.e., the demand for a particular service does not depend on the prices of the other services; (iii) there is no income (or wealth) effect for the requested services, that is, the demand for a particular service does not depend on the user's income. This can be justified as follows. Since communication services is just one good among others that the user is willing to buy, then because one extra unit of wealth will spread itself around, the wealth effect for that good is bound to be small. The assumption that there is no income effect implies that the Walrasian and Hicksian demand functions are identical [19]. This in turn implies that the demand is a decreasing function of the price (Compensated Law of Demand). Assumption (A9) implies that the network does not have to know the duration of any of the requests it receives. Assumption (A10) ensures that services that have been admitted at some allocation instant and have not been completed over the next $\widehat{T}$ units of time, continue to be served without any violation of their QoS requirements, until they inform the network that they have been completed. Finally assumption (A11) does not allow any statistical multiplexing of resources among different connections at ATM switches. An assumption more realistic than (A11) would allow resource sharing among different connections at ATM switches. The implications of this assumption will be further discussed in Section 5. Weakening assumptions (A5) and/or (A11), as discussed above, would result in a problem considerably more difficult than the one we have formulated in this paper. Such a problem will be the topic of future research. 
Let $\mu_{i}^{s}, \beta_{i}^{s 1}$ be the bandwidth and buffer allocation, respectively, for a type $s$ service over the $i$ th link along its route $r_{s}=\left\{1,2, \ldots, P_{s}\right\}$. Note that for $l \notin r_{s}, \mu_{l s}=\beta_{l s}=0$. We define by $M_{s}\left\{\mu_{0}^{s}, b_{s}(\cdot)\right\}$ the set of all type $s$ messages that are conformant, i.e., they satisfy Eqs (2.2) and (2.3). Let $x_{s}$ be the amount of a type $s$ service that is provided by the network and $w_{s}$ be the corresponding price. Then the service allocation decision:

$$
(\boldsymbol{x}, \boldsymbol{w}, \boldsymbol{\mu}, \boldsymbol{\beta}) \triangleq\left\{x_{s}, w_{s},\left\{\mu_{i}^{s}, \beta_{i}^{s}\right\}_{i \in r_{s}}\right\}_{s \in S}
$$

is conformant, if it satisfies the following equations:

$$
\begin{aligned}
& 0 \leqslant x_{s} \leqslant R_{s}\left(w_{s}\right), \quad \forall s \in S, \\
& w_{s} \geqslant 0, \quad \forall s \in S, \\
& \sum_{s \in S} x_{s} \mu_{l}^{s} \leqslant C_{l}(k \widehat{T}) \quad \text { and } \sum_{s \in S} x_{s} \beta_{l}^{s} \leqslant B_{l}(k \widehat{T}), \quad \forall l \in L, \\
& \mathcal{L}_{m}(\boldsymbol{\mu}, \boldsymbol{\beta}) \leqslant L_{s}, \quad \forall m(\cdot) \in M_{s}\left\{\mu_{0}^{s}, b_{s}(\cdot)\right\}, \forall s \in S, \\
& \mathcal{D}_{m}(\boldsymbol{\mu}, \boldsymbol{\beta}) \leqslant D_{s}, \quad \forall m(\cdot) \in M_{s}\left\{\mu_{0}^{s}, b_{s}(\cdot)\right\}, \forall s \in S,
\end{aligned}
$$

where: $C_{l}(k \widehat{T}), B_{l}(k \widehat{T})$ are the bandwidth and buffers, respectively, that are available for allocation at the beginning of the $k$ th allocation period at node $l \in L^{2} ; \mathcal{L}_{m}(\boldsymbol{\mu}, \boldsymbol{\beta})$ is the percentage of cell loss and $\mathcal{D}_{m}(\boldsymbol{\mu}, \boldsymbol{\beta})$ is the maximum end-to-end cell delay (in units of time) for message $m(\cdot)$, resulting from the allocation $(\boldsymbol{x}, \boldsymbol{w}, \boldsymbol{\mu}, \boldsymbol{\beta})$.

The first two inequalities (2.4), (2.5) state that the supply should not be greater than the demand and also that the number of offered services and their corresponding prices should not be negative quantities. The next family of inequalities (2.6) states that the allocation should not exceed the available resources. Finally (2.7) and (2.8) guarantee that the offered services satisfy the quality requirements.

Based on the above assumptions and discussion we now propose the following two-step approach to formulating the service provisioning problem.

Step 1: Find a family of functions $\widehat{\mathcal{L}}_{s}(\boldsymbol{\mu}, \boldsymbol{\beta})$ and $\widehat{\mathcal{D}}_{s}(\boldsymbol{\mu}, \boldsymbol{\beta}), \forall s \in S$ such that,

$$
\begin{aligned}
& \mathcal{L}_{m}(\boldsymbol{\mu}, \boldsymbol{\beta}) \leqslant \widehat{\mathcal{L}}_{s}(\boldsymbol{\mu}, \boldsymbol{\beta}) \text { and } \\
& \mathcal{D}_{m}(\boldsymbol{\mu}, \boldsymbol{\beta}) \leqslant \widehat{\mathcal{D}}_{s}(\boldsymbol{\mu}, \boldsymbol{\beta}),
\end{aligned}
$$

for all $m(\cdot) \in M_{s}\left\{\mu_{0}^{s}, b_{s}(\cdot)\right\}$ and for all $s \in S$.

Step 2: Solve the following optimization problem:

Maximize the welfare function,

$$
W(\boldsymbol{x}, \boldsymbol{w}, \boldsymbol{\mu}, \boldsymbol{\beta}) \triangleq \sum_{s \in S} x_{s} w_{s}+\sum_{s \in S} \int_{w_{s}}^{\infty} \min \left(x_{s}, R_{s}(u)\right) \mathrm{d} u
$$

subject to conditions (2.4)-(2.6) and (2.12) which is given below:

$$
\widehat{\mathcal{L}}_{s}(\boldsymbol{\mu}, \boldsymbol{\beta}) \leqslant L_{s} \quad \text { and } \quad \widehat{\mathcal{D}}_{s}(\boldsymbol{\mu}, \boldsymbol{\beta}) \leqslant D_{s}, \quad \forall s \in S .
$$

\footnotetext{
${ }^{1}$ The letter $l$, when used as a subscript instead of $i$, is an index over the set $L$ of all links of the network.

${ }^{2}$ This is consistent with assumption (A10). Note that $C_{l}(0)=C_{l}$ and $B_{l}(0)=B_{l}$.
} 
The first term of the welfare function (Eq. (2.11)), is the network revenue and the second is the user surplus [23].

We now discuss the features of our two step approach to the service provisioning problem. Ideally we would like to solve the problem of maximizing (2.11) over the set of all conformant allocations (i.e., those that satisfy (2.4)-(2.8)). We don't do that. Instead, in Step 2, we replace (2.7) and (2.8) by (2.12). The consequence of that is the following: If the upper bounds in Step 1 are tight then the solutions of the two problems are the same. If not, then the set of all conformant allocations is a superset of the allocations that also satisfy (2.12). Therefore the two problems may not have the same solution. As we will see in Section 3, where we compute the upper bounds for Step 1, these bounds are not tight. Therefore, in our service provisioning approach the optimization is done over a subset of all possible conformant service allocation decisions. Nevertheless, our approach to service provisioning explicitly incorporates QoS requirements that capture the interaction of resource allocation (bandwidth and buffers), percentage of cell loss and end-to-end cell delay.

We present the solution of Step 1 in the next section. The solution of the optimization problem in Step 2, is given in Section 4.

\section{Quality of service requirements}

In this section we consider a type $s$ service provided by a connection over the fixed route $r_{s}=\left\{1,2, \ldots, P_{s}\right\}$, and the set $M_{s}\left\{\mu_{0}^{s}, b_{s}(\cdot)\right\}$ of all type $s$ messages. Given the fixed allocation $\left\{\mu_{i}^{s}, \beta_{i}^{s}\right\}_{i \in r_{s}}$ along the route, we want to determine upper bounds for the percentage of cell loss and the end-to-end cell delay, over all messages in $M_{s}\left\{\mu_{0}^{s}, b_{s}(\cdot)\right\}$. For convenience we omit the subscript (superscript) $s$. Furthermore, we denote by $(\boldsymbol{\mu}, \boldsymbol{\beta})$ the fixed allocation $\left\{\mu_{k}, \beta_{k}\right\}_{k=1}^{P}$ along the route.

\subsection{An upper bound on the percentage of cell loss along a fixed route}

Consider the multinode system of Fig. 2, and assume that the input message $m_{0}(\cdot)$ belongs to the family $M\left\{\mu_{0}, b(\cdot)\right\}$. We denote by $m_{k}(\cdot)$ the resulting message at the output of node $k, 1 \leqslant k \leqslant P$. Let $T_{m_{k}}$ be the duration (in units of time) and $V_{m_{k}}$ the volume (in cells) of message $m_{k}(\cdot), 0 \leqslant k \leqslant P$.

Without any loss of optimality we can restrict attention to allocations that satisfy,

$$
\begin{aligned}
& \mu_{k} \leqslant \mu_{k-1}, \quad 1 \leqslant k \leqslant P \\
& \beta_{1} \leqslant \min \left(b\left(\mu_{1}\right), \widehat{T}\left(\mu_{0}-\mu_{1}\right)\right), \\
& \beta_{k} \leqslant \widehat{T}_{k-1}\left(\mu_{k-1}-\mu_{k}\right), \quad 2 \leqslant k \leqslant P,
\end{aligned}
$$

where $\widehat{T}_{k}$ is an upper bound on $T_{m_{k}}$. This is because if (3.1) is not satisfied at some node $k$, part of the resources allocated to that node is never utilized. To find an upper bound on $T_{m_{k}}$ we notice the following:

$$
T_{m_{0}} \leqslant \widehat{T}, \quad \text { and } \quad T_{m_{k}} \leqslant T_{m_{k-1}}+\frac{\beta_{k}}{\mu_{k}}, \quad 1 \leqslant k \leqslant P-1 .
$$

Therefore,

$$
\widehat{T}_{k}=\widehat{T}+\sum_{j=1}^{k} \frac{\beta_{j}}{\mu_{j}}, \quad 1 \leqslant k \leqslant P-1 .
$$

The main problem that we want to solve in this section is the following:

For a fixed resource allocation $(\boldsymbol{\mu}, \boldsymbol{\beta})$ that satisfies $(3.1)$, find a function $\widehat{\mathcal{L}}(\boldsymbol{\mu}, \boldsymbol{\beta})$ such that:

$$
\mathcal{L}_{m_{0}}(\boldsymbol{\mu}, \boldsymbol{\beta}) \leqslant \widehat{\mathcal{L}}(\boldsymbol{\mu}, \boldsymbol{\beta}), \quad \forall m_{0}(\cdot) \in M\left\{\mu_{0}, b(\cdot)\right\}
$$




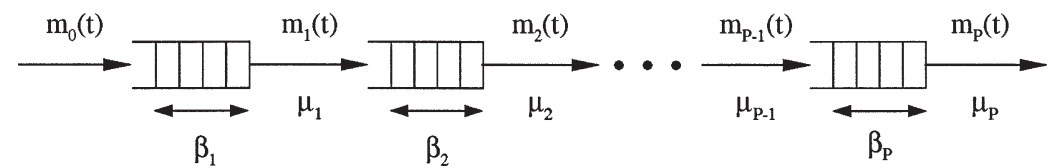

Fig. 2. Connection spanning $P$ nodes in sequence.

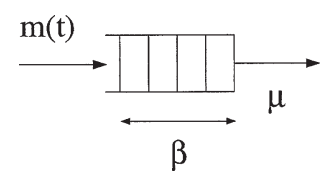

Fig. 3. Single node model.

where $\mathcal{L}_{m_{0}}(\boldsymbol{\mu}, \boldsymbol{\beta})$ is the percentage of cell loss for message $m_{0}(\cdot)$ when it travels through the multinode system of Fig. 2.

The percentage of cell loss $\mathcal{L}_{m_{0}}^{k}(\boldsymbol{\mu}, \boldsymbol{\beta})$ at each individual node due to $m_{0}(\cdot)$ is,

$$
\mathcal{L}_{m_{0}}^{k}(\boldsymbol{\mu}, \boldsymbol{\beta})=1-\frac{V_{m_{k}}}{V_{m_{k-1}}}, \quad 1 \leqslant k \leqslant P,
$$

whereas the percentage of cell loss along the route is,

$$
\mathcal{L}_{m_{0}}(\boldsymbol{\mu}, \boldsymbol{\beta})=1-\frac{V_{m_{p}}}{V_{m_{0}}}=1-\prod_{k=1}^{P} \frac{V_{m_{k}}}{V_{m_{k-1}}}=1-\prod_{k=1}^{P}\left(1-\mathcal{L}_{m_{0}}^{k}(\boldsymbol{\mu}, \boldsymbol{\beta})\right) .
$$

To find an upper bound on $\mathcal{L}_{m_{0}}(\boldsymbol{\mu}, \boldsymbol{\beta})$ we solve two problems that refer to a single server with a finite buffer $\beta$ and a maximum service rate $\mu$, as shown in Fig. 3 .

In the first problem $(\mathrm{P} 1)$ we find a message $\widehat{m}_{0}(\cdot)$ that satisfies the following inequality,

$$
(\mathrm{P} 1): \mathcal{L}_{\widehat{m}_{0}}(\mu, \beta) \geqslant \max _{m(\cdot) \in M\left\{\mu_{0}, b(\cdot)\right\}} \mathcal{L}_{m}(\mu, \beta),
$$

where $\mathcal{L}_{m}(\mu, \beta)$ denotes the percentage of cell loss for message $m(\cdot)$ as it passes through the single node system. This result establishes the fact that,

$$
\mathcal{L}_{m_{0}}^{1}(\boldsymbol{\mu}, \boldsymbol{\beta}) \leqslant \mathcal{L}_{\widehat{m}_{0}}\left(\mu_{1}, \beta_{1}\right), \quad \forall m_{0}(\cdot) \in M\left\{\mu_{0}, b(\cdot)\right\} .
$$

In the second problem (P2) we consider the family $M^{\prime}\left\{\mu_{0}\right\}$ of input messages with finite duration $T$ and maximum input rate $\mu_{0}$. That is,

$$
M^{\prime}\left\{\mu_{0}\right\} \triangleq\left\{m(\cdot), m(t) \leqslant \mu_{0}, 0 \leqslant t \leqslant T\right\} .
$$

Then we prove that there exists a message $\widehat{m}_{0}^{\prime}(\cdot) \in M^{\prime}\left\{\mu_{0}\right\}$ such that,

$$
\text { (P2): } \widehat{m}_{0}^{\prime}(\cdot)=\arg \max _{m(\cdot) \in M^{\prime}\left\{\mu_{0}\right\}} \mathcal{L}_{m}(\mu, \beta),
$$

where $\mathcal{L}_{m}(\mu, \beta)$ denotes, as before, the percentage of cell loss for message $m(\cdot)$.

Since for every $m_{0}(\cdot) \in M\left\{\mu_{0}, b(\cdot)\right\}$ we have that $m_{k}(\cdot) \in M^{\prime}\left\{\mu_{k}\right\}, 1 \leqslant k \leqslant P-1$, the following set of inequalities is true,

$$
\mathcal{L}_{m_{0}}^{k}(\boldsymbol{\mu}, \boldsymbol{\beta}) \leqslant \mathcal{L}_{\widehat{m}_{k-1}^{\prime}}\left(\mu_{k}, \beta_{k}\right), \quad \forall m_{0}(\cdot) \in M\left\{\mu_{0}, b(\cdot)\right\}, \quad 2 \leqslant k \leqslant P,
$$


where,

$$
\widehat{m}_{k-1}^{\prime} \triangleq \arg \max _{m(\cdot) \in M^{\prime}\left\{\mu_{k-1}\right\}} \mathcal{L}_{m}\left(\mu_{k}, \beta_{k}\right), \quad 2 \leqslant k \leqslant P .
$$

Equations (3.3), (3.5) and (3.7) establish the fact that,

$$
\mathcal{L}_{m_{0}}(\boldsymbol{\mu}, \boldsymbol{\beta}) \leqslant \widehat{\mathcal{L}}(\boldsymbol{\mu}, \boldsymbol{\beta}), \quad \forall m_{0}(\cdot) \in M\left\{\mu_{0}, b(\cdot)\right\},
$$

where,

$$
\widehat{\mathcal{L}}(\boldsymbol{\mu}, \boldsymbol{\beta})=1-\left(1-\mathcal{L}_{\widehat{m}_{0}}\left(\mu_{1}, \beta_{1}\right)\right) \prod_{k=2}^{P}\left(1-\mathcal{L}_{\widehat{m}_{k-1}^{\prime}}\left(\mu_{k}, \beta_{k}\right)\right) .
$$

We now proceed in the formulation and solution of problem (P1).

\subsubsection{The problem in the first node: formulation and solution of (P1)}

Let the single node model of Fig. 3. We consider this system to be a work conserving server with finite buffer $\beta$ and maximum service rate $\mu$.

We consider input messages $m(\cdot)$ that belong to the family $M\left\{\mu_{0}, b(\cdot)\right\}$ and have duration $\widehat{T}$. This is in accordance with assumptions (A3), (A4) and (A5). Since for $\mu \geqslant \mu_{0}$ there are no cell losses we also make the assumption that $\mu<\mu_{0}$. Finally we assume that the buffer occupancy at the beginning of the transmission session is zero.

Let $\mathcal{L}_{m}(\mu, \beta)$ denote the percentage of cell loss for message $m(\cdot)$ as it passes through the single node system. We also denote by $\mathcal{E}_{m}(\mu, \beta)$ the corresponding number of cells lost. We want to solve the following problem: (P1) Determine an input message $\widehat{m}_{0}(\cdot)$ such that,

$$
\mathcal{L}_{m}(\mu, \beta) \leqslant \mathcal{L}_{\widehat{m}_{0}}(\mu, \beta), \quad \forall m(\cdot) \in M\left\{\mu_{0}, b(\cdot)\right\} .
$$

The main result of Section 3.1.1 is summarized by the following theorem:

Theorem 3.1. Let $\widehat{N}$ be the largest integer such that

$$
\widehat{N} \frac{b(\mu) \mu_{0}}{\mu\left(\mu_{0}-\mu\right)}+\frac{b(\mu)}{\mu_{0}-\mu} \leqslant \widehat{T},
$$

and let

$$
\widehat{r} \triangleq \widehat{T}-\widehat{N} \frac{b(\mu) \mu_{0}}{\mu\left(\mu_{0}-\mu\right)}-\frac{b(\mu)}{\mu_{0}-\mu} .
$$

Then,

$$
\mathcal{L}_{m}(\mu, \beta) \leqslant \mathcal{L}_{\widehat{m}_{0}}(\mu, \beta), \quad \forall m(\cdot) \in M\left\{\mu_{0}, b(\cdot)\right\},
$$

where $\widehat{m}_{0}(\cdot)$ has the following characteristics (see Fig. 4):

(i) It satisfies the burstiness constraint at the rate $\mu$.

(ii) It consists of $\widehat{N}+2$ 'ON' intervals, during which $\widehat{m}_{0}(t)=\mu_{0}$, and $\widehat{N}+1$ 'OFF' intervals, during which $\widehat{m}_{0}(t)=0$. The duration of each 'ON' interval is equal to $b(\mu) /\left(\mu_{0}-\mu\right)$ and the duration of each 'OFF' interval is equal to $b(\mu) / \mu$.

(iii) If $\widehat{r}>\beta \mu_{0} /\left(\mu\left(\mu_{0}-\mu\right)\right)$, then $\widehat{m}_{0}(\cdot)$ ends with an 'ON' interval of duration $\alpha /\left(\mu_{0}-\mu\right)$ preceded by an 'OFF' interval of duration $\alpha / \mu$, where $\alpha \triangleq \widehat{r} \mu\left(\mu_{0}-\mu\right) / \mu_{0}$. If $\widehat{r} \leqslant \beta \mu_{0} /\left(\mu\left(\mu_{0}-\mu\right)\right)$ then $\widehat{m}_{0}(\cdot)$ ends with an 'OFF' interval of duration $\widehat{r}$. 


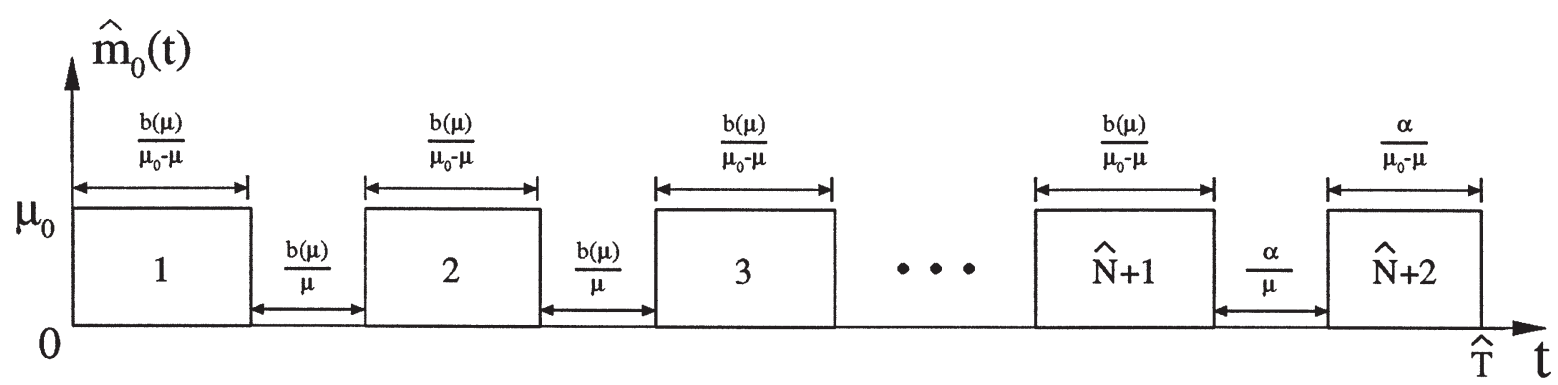

Fig. 4. Maximum cell loss message.

The message $\widehat{m}_{0}(\cdot)$ described above has the following additional property:

$$
\mathcal{E}_{m}(\mu, \beta) \leqslant \mathcal{E}_{\widehat{m}_{0}}(\mu, \beta), \quad \forall m(\cdot) \in M\left\{\mu_{0}, b(\cdot)\right\} .
$$

Discussion. The intuition behind the result presented in the theorem is the following. The requirement that the message, that provides the upper bound on the percentage of cell loss, satisfies the burstiness constraint at rate $\mu$ leads us to consider a fictitious buffer with capacity $b(\mu)$ and maximum service rate $\mu$. This fictitious buffer should never overflow by any message that satisfies the burstiness constraint at rate $\mu$. The message that incurs the maximum percentage of cell loss must fill in the fictitious buffer as quickly as possible and then empty it as quickly as possible. As soon as the buffer becomes empty the message should start refilling it again with the maximum rate. This pattern must continue through out the whole duration of the message. All these considerations lead to the 'ON-OFF' 3 message that is described in the statement of the theorem. We prove that this message indeed provides an upper bound on the percentage of cell loss. The main idea of the proof is the following. We select an arbitrary input message $m(\cdot) \in M\left\{\mu_{0}, b(\cdot)\right\}$, and starting from $m(\cdot)$, we construct a sequence of messages with the following feature: each of these messages results from a simple modification of its precedent in the sequence; each resulting message satisfies the burstiness constraint at the rate $\mu$; and each message incurs an equal or higher percentage of cell loss than its precedent. The construction of the abovementioned sequence of messages is described in a series of lemmas. The intuition behind each lemma is discussed right after their statement.

We define the following quantities to be used in the proof of the theorem:

$b_{m}^{r}(t)$ : buffer occupancy (in cells) at time $t$, due to message $m(\cdot)$,

$b_{m}^{f}(t)$ : buffer occupancy of the fictitious buffer at time $t$, due to message $m(\cdot)$,

$X_{\mathrm{ON}}$ : duration of an 'ON' period of an ON-OFF message,

$X_{\mathrm{OFF}}$ : duration of an 'OFF' period of an ON-OFF message.

We also make the assumption that both the 'real' and the fictitious buffer are empty at time $t=0$, that is: $b_{m}^{r}(0)=b_{m}^{f}(0)=0$.

We now proceed to the series of lemmas that establish the result of Theorem 3.1.

Lemma 3.1. We can replace any message $m(\cdot) \in M\left\{\mu_{0}, b(\cdot)\right\}$, that has strictly positive losses, by an ON-OFF message $m_{1}(\cdot)$ that satisfies the burstiness constraint at rate $\mu$ and has the same number of cell losses and a greater or equal percentage of loss than the original. Furthermore, $m_{1}(\cdot)$ is such that,on every ON interval it has strictly positive losses and $X_{\mathrm{OFF}} \geqslant \beta / \mu$.

\footnotetext{
${ }^{3}$ That is a message with rate that takes only two values, either zero or the maximum.
} 
Discussion. The intuition behind this lemma is the following. An ON-OFF message that satisfies the burstiness constraint at rate $\mu$ can achieve the same cell loss as any other message $m(\cdot)$ that satisfies the burstiness constraint at rate $\mu$, in less time and with the minimum volume. Indeed there is a minimum volume required to fill up the buffer the first time and also between successive overflows. Any extra volume that does not contribute to the cell loss can therefore be discarded.

Proof. Let $m(\cdot)$ be any message in $M\left\{\mu_{0}, b(\cdot)\right\}$ with strictly positive losses. We can then use the representation proposed in [26], to find $\mathcal{E}_{m}(\mu, \beta)$, i.e., the number of cells dropped as a result of buffer overflow during the transmission of that message. That is,

$$
\mathcal{E}_{m}(\mu, \beta)=\sum_{j=1}^{N}\left(\int_{\tau_{j}}^{s_{j}}(m(r)-\mu) \mathrm{d} r-\beta\right)
$$

where $N>0$ is finite and $0 \leqslant \tau_{1}<s_{1}<\cdots<\tau_{N}<s_{N} \leqslant \widehat{T}$.

Although $N$ and $\left\{\tau_{i}, s_{i}\right\}, i=1, \ldots, N$, are not unique, we can define them as follows (see Fig. 5):

$N \triangleq$ number of times the buffer overflows, under the condition that the buffer becomes empty between successive overflows (e.g., for the realization given in Fig. 5, $N=3$ ),

$\tau_{i} \triangleq$ time instant the buffer leaves the 'empty' state (i.e., $\left.b_{m}^{r}\left(\tau_{i}\right)=0\right)$ and reaches the 'full' state (i.e., $\left.b_{m}^{r}\left(\tau_{i}^{\prime}\right)=\beta, \tau_{i}^{\prime}>\tau_{i}\right)$, before entering the 'empty' state again (i.e., $b_{m}^{r}(t) \neq 0, t \in\left(\tau_{i}, \tau_{i}^{\prime}\right)$ ),

$s_{i} \triangleq$ time instant that the buffer leaves the 'full' state (i.e., $b_{m}^{r}\left(s_{i}\right)=\beta$ ) and reaches the 'empty' state (i.e., $\left.b_{m}^{r}\left(s_{i}^{\prime}\right)=0, s_{i}^{\prime}>s_{i}\right)$, before entering the 'full' state again (i.e., $\left.b_{m}^{r}(t) \neq \beta, t \in\left(s_{i}, s_{i}^{\prime}\right)\right)$.

We also define $E_{i}$ to be the number of cells lost during the interval $\left[\tau_{i}, s_{i}\right]$, that is,

$$
E_{i} \triangleq \int_{\tau_{i}}^{s_{i}}(m(r)-\mu) \mathrm{d} r-\beta .
$$

We now construct the ON-OFF message $m_{1}(\cdot)$ as follows:

$$
\hat{t}_{1} \triangleq 0, \quad \hat{t}_{1}^{\prime} \triangleq \frac{\beta+E_{1}}{\mu_{0}-\mu}, \quad \hat{t}_{i} \triangleq \hat{t}_{i-1}^{\prime}+\tau_{i}-s_{i-1}, \quad \hat{t}_{i}^{\prime} \triangleq \hat{t}_{i}+\frac{\beta+E_{i}}{\mu_{0}-\mu}, \quad i=2, \ldots, N,
$$

where $\hat{t}_{i}$ is the time instant that the $i$ th ON interval starts, and $\hat{t}_{i}^{\prime}$ is the time instant that the $i$ th ON interval ends (Fig. 6).

By construction, message $m_{1}(\cdot)$ incurs positive losses on every $\mathrm{ON}$ interval. The remainder of the proof proceeds in four steps.

Step I. We show that $X_{\mathrm{OFF}} \geqslant \beta / \mu$.

By assumption $\tau_{i}>s_{i-1}$. Hence,

$$
\begin{aligned}
b_{m}^{r}\left(\tau_{i}\right) & =b_{m}^{r}\left(s_{i-1}\right)+\int_{s_{i-1}}^{\tau_{i}}(m(r)-\mu) \mathrm{d} r \Rightarrow \int_{s_{i-1}}^{\tau_{i}} m(r) \mathrm{d} r+\beta=\mu\left(\tau_{i}-s_{i-1}\right) \\
& \Rightarrow \tau_{i}-s_{i-1} \geqslant \beta / \mu \Rightarrow \hat{t}_{i}-\hat{t}_{i-1}^{\prime} \geqslant \beta / \mu .
\end{aligned}
$$

The second equality is true because by definition $b_{m}^{r}\left(\tau_{i}\right)=0, b_{m}^{r}\left(s_{i-1}\right)=\beta$. The first inequality is true because $m(r) \geqslant 0$ and the last one because of (3.13).

Step II. We show that the duration of $m_{1}(\cdot)$ is shorter than that of $m(\cdot)$.

By definition,

$$
E_{i}=\int_{\tau_{i}}^{s_{i}}(m(r)-\mu) \mathrm{d} r-\beta
$$




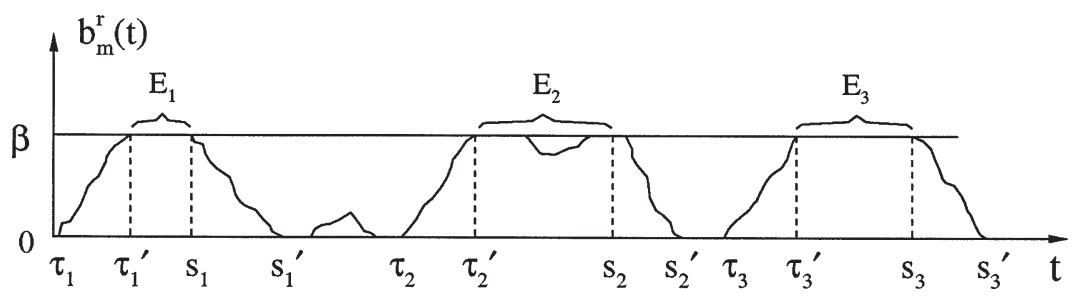

Fig. 5. Example of a buffer occupancy realization.

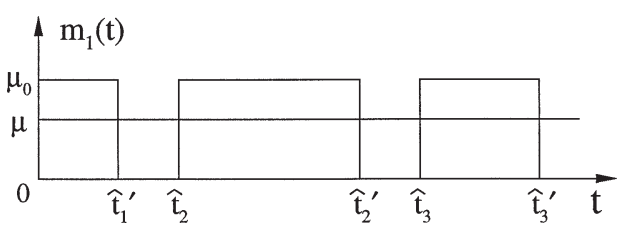

Fig. 6. ON-OFF message.

Hence,

$$
E_{i}+\beta \leqslant \int_{\tau_{i}}^{s_{i}}\left(\mu_{0}-\mu\right) \mathrm{d} r \Rightarrow \frac{\beta+E_{i}}{\mu_{0}-\mu} \leqslant s_{i}-\tau_{i} \Rightarrow \hat{t}_{i}^{\prime}-\hat{t}_{i} \leqslant s_{i}-\tau_{i} .
$$

The first inequality is true because $m(r) \leqslant \mu_{0}$ and the third inequality because of (3.13). Furthermore,

$$
\hat{t}_{N}^{\prime}=\sum_{i=2}^{N}\left(\hat{t}_{i}-\hat{t}_{i-1}^{\prime}\right)+\sum_{i=1}^{N}\left(\hat{t}_{i}^{\prime}-\hat{t}_{i}\right) \leqslant \sum_{i=2}^{N}\left(\tau_{i}-s_{i-1}\right)+\sum_{i=1}^{N}\left(s_{i}-\tau_{i}\right)=s_{N}-\tau_{1} \leqslant s_{N} .
$$

The first inequality is true because of (3.13) and (3.14), and the last one because $\tau_{1} \geqslant 0$.

Step III. We show that message $m_{1}(\cdot)$ has the same losses as $m(\cdot)$ and a greater or equal percentage of loss. The cell loss of message $m_{1}(\cdot)$ is,

$$
\mathcal{E}_{m_{1}}(\mu, \beta)=\sum_{i=1}^{N}\left(\left(\mu_{0}-\mu\right)\left(\hat{t}_{i}^{\prime}-\hat{t}_{i}\right)-\beta\right)=\sum_{i=1}^{N} E_{i}=\mathcal{E}_{m}(\mu, \beta),
$$

where the first equality is true because, according to Step I, $b^{r}\left(\hat{t}_{i}\right)=0$, the second is true because of (3.13), and the last one because of (3.12). This establishes the fact that messages $m(\cdot)$ and $m_{1}(\cdot)$ incur the same losses.

From the construction of message $m_{1}(\cdot)$ (Eq. (3.13)) and the definition of $E_{i}$ we have that,

$$
\left(\mu_{0}-\mu\right)\left(\hat{t}_{i}^{\prime}-\hat{t}_{i}\right)=\beta+E_{i}=\int_{\tau_{i}}^{s_{i}}(m(r)-\mu) \mathrm{d} r
$$

Therefore,

$$
\mu_{0}\left(\hat{t}_{i}^{\prime}-\hat{t}_{i}\right)=\int_{\tau_{i}}^{s_{i}} m(r) \mathrm{d} r+\mu\left(\left(\hat{t}_{i}^{\prime}-\hat{t}_{i}\right)-\left(s_{i}-\tau_{i}\right)\right) .
$$

Since by (3.14), $s_{i}-\tau_{i} \geqslant \hat{t}_{i}^{\prime}-\hat{t}_{i}$, it follows from (3.15) that,

$$
\int_{\tau_{i}}^{s_{i}} m(r) \mathrm{d} r \geqslant \mu_{0}\left(\hat{t}_{i}^{\prime}-\hat{t}_{i}\right), \quad 1 \leqslant i \leqslant N .
$$


This implies that,

$$
\int_{0}^{\widehat{T}} m_{1}(r) \mathrm{d} r=\sum_{i=1}^{N} \mu_{0}\left(\hat{t}_{i}^{\prime}-\hat{t}_{i}\right) \leqslant \sum_{i=1}^{N} \int_{\tau_{i}}^{s_{i}} m(r) \mathrm{d} r \leqslant \int_{0}^{\widehat{T}} m(r) \mathrm{d} r .
$$

Therefore the volume of message $m(\cdot)$ is at least as much as the volume of message $m_{1}(\cdot)$. Since, by the previous argument, they both have the same losses, it follows that the percentage of loss for $m_{1}(\cdot)$ is at least as much as the percentage of loss for $m(\cdot)$, that is,

$$
\mathcal{L}_{m_{1}}(\mu, \beta) \geqslant \mathcal{L}_{m}(\mu, \beta) .
$$

Step IV. We show that $m_{1}(\cdot)$ satisfies the burstiness constraint at the rate $\mu$.

For any $j \in\{1, \ldots, N\}$ we have:

$$
\begin{aligned}
b_{m_{1}}^{f}\left(\hat{t}_{j}^{\prime}\right) & =\sum_{i=1}^{j}\left(\mu_{0}-\mu\right)\left(\hat{t}_{i}^{\prime}-\hat{t}_{i}\right)-\sum_{i=2}^{j} \mu\left(\hat{t}_{i}-\hat{t}_{i-1}^{\prime}\right)=\sum_{i=1}^{j}\left(\beta+E_{i}\right)-\sum_{i=2}^{j} \mu\left(\tau_{i}-s_{i-1}\right) \\
& =\sum_{i=1}^{j} \int_{\tau_{i}}^{s_{i}}(m(r)-\mu) \mathrm{d} r-\sum_{i=2}^{j} \mu\left(\tau_{i}-s_{i-1}\right) \leqslant \int_{\tau_{1}}^{s_{j}}(m(r)-\mu) \mathrm{d} r=b_{m}^{f}\left(s_{j}\right),
\end{aligned}
$$

where the second equality is true because of (3.13), the third equality is true because of the definition of $E_{i}$, the inequality holds because $m(r) \geqslant 0$ and the last equality holds because $b_{m}^{f}(0)=b_{m}^{f}\left(\tau_{1}\right)=0$. Since $m(\cdot) \in M\left\{\mu_{0}, b(\cdot)\right\}$ we have that $b_{m}^{f}\left(s_{j}\right) \leqslant b(\mu)$ and therefore from (3.17), $b_{m_{1}}^{f}\left(\hat{t}_{j}^{\prime}\right) \leqslant b(\mu)$. This implies that $b_{m_{1}}^{f}(t) \leqslant b(\mu), \forall t \geqslant 0$.

The proof of Lemma 3.1 is complete.

Lemma 3.2. We can replace any segment $s_{1}(\cdot)$ of $m(\cdot)$, that consists of two consecutive ON-OFF intervals, where the OFF-intervals have duration $X_{\mathrm{OFF}} \geqslant \beta / \mu$, by another segment $\widehat{s}_{1}(\cdot)$, that consists of either a single or a double ON-OFF interval with the following characteristics: In both cases the OFF intervals have length $\widehat{X}_{\mathrm{OFF}} \geqslant \beta / \mu$, while in the second case, we have that: $b_{\widehat{s}_{1}}^{f}\left(\hat{t}_{1}^{\prime}\right)=b_{\widehat{s}_{1}}^{f}\left(\hat{t}_{2}^{\prime}\right)=b(\mu)$, where $\hat{t}_{i}^{\prime}, i=1,2$, is the time instant where the ith ON interval ends. The resulting message $m_{2}(\cdot)$, satisfies the burstiness constraint at rate $\mu$, it is shorter in duration than $m(\cdot)$, and it has at least the same losses and a greater or equal percentage of loss than the original.

Discussion. The intuition behind this lemma is that, given an ON-OFF message that satisfies the burstiness constraint at rate $\mu$, we can combine ON periods to form ON periods that bring the fictitious buffer to its maximum occupancy.

Proof. Consider the segment $s_{1}(\cdot)$ of $m(\cdot)$, shown in Fig. 7, where we assume that $x_{1}^{\prime} \geqslant \beta / \mu$ and $x_{2}^{\prime} \geqslant \beta / \mu$, and therefore that $b_{m}^{r}\left(t_{2}\right)=b_{m}^{r}\left(t_{3}\right)=0$.

Define,

$$
\alpha \triangleq b_{m}^{f}\left(t_{1}\right)+\left(x_{1}+x_{2}\right)\left(\mu_{0}-\mu\right)-b(\mu) .
$$

Since $m(\cdot) \in M\left\{\mu_{0}, b(\cdot)\right\}$,

$$
b_{m}^{f}\left(t_{2}^{\prime}\right) \leqslant b(\mu) .
$$

Consequently,

$$
b_{m}^{f}\left(t_{1}\right)+\left(x_{1}+x_{2}\right)\left(\mu_{0}-\mu\right)-x_{1}^{\prime} \mu \leqslant b(\mu),
$$


or equivalently,

$$
x_{1}^{\prime}-\frac{\alpha}{\mu} \geqslant 0,
$$

where the last inequality is true because of (3.18).

If $\alpha<\beta$, consider the modified message segment $\widehat{s}_{1}(\cdot)$ of Fig. 8 , where

$$
\widehat{x}_{1} \triangleq x_{1}+x_{2}-\frac{\alpha^{+}}{\mu_{0}-\mu}, \quad \widehat{x}_{1}^{\prime} \triangleq x_{1}^{\prime}+x_{2}^{\prime}-\frac{\alpha^{+}}{\mu}, \quad \alpha^{+} \triangleq \max (0, \alpha)
$$

while if $\alpha \geqslant \beta$, consider the segment shown in Fig. 9, where

$$
\widehat{x}_{1} \triangleq x_{1}+x_{2}-\frac{\alpha}{\mu_{0}-\mu}, \quad \widehat{x}_{1}^{\prime} \triangleq \frac{\alpha}{\mu}, \quad \widehat{x}_{2} \triangleq \frac{\alpha}{\mu_{0}-\mu}, \quad \widehat{x}_{2}^{\prime} \triangleq x_{1}^{\prime}+x_{2}^{\prime}-\frac{\alpha}{\mu} .
$$

In both cases we have by construction of the new message $m_{2}(\cdot)$ that

$$
b_{m_{2}}^{f}\left(t_{1}\right)=b_{m}^{f}\left(t_{1}\right) \quad \text { and } \quad b_{m_{2}}^{r}\left(t_{1}\right)=b_{m}^{r}\left(t_{1}\right) .
$$

Our goal is to show that for the two cases above the new segment $\widehat{s}_{1}(\cdot)$ satisfies the burstiness constraint at rate $\mu$, has at least the same losses as the original, less or equal volume, smaller duration, and brings the system at the same buffer occupancy (both for the real and the fictitious buffer). That would prove that by substituting any 'type- $s_{1}(\cdot)$ ' segment in $m(\cdot)$ by a 'type- $\widehat{s}_{1}(\cdot)$ ' segment, the resulting message $m_{2}(\cdot)$ : (i) satisfies the burstiness constraint at rate $\mu$; (ii) has less or equal volume than the original; (iii) it has greater or equal losses (since the loss for the other part of the message has not changed). Therefore the number of cells lost and the percentage of cell loss are at least as much as for the original message $m(\cdot)$.

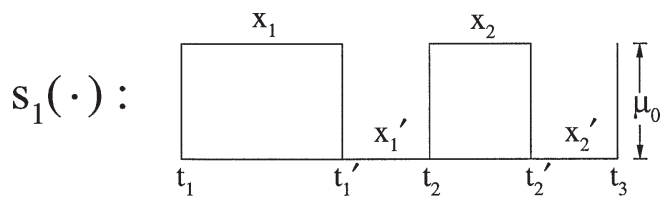

Fig. 7. Original message segment.

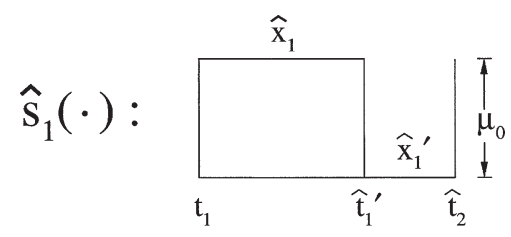

Fig. 8. Modified message segment when $\alpha<\beta$.

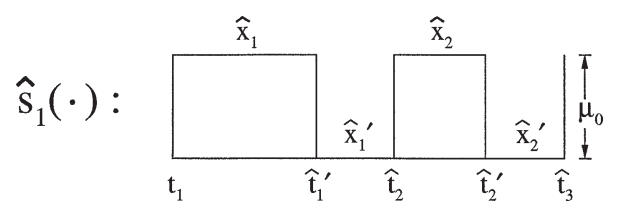

Fig. 9. Modified message segment when $\alpha \geqslant \beta$. 
The proof of each of the two cases $(\alpha<\beta, \alpha \geqslant \beta)$ consists of five steps.

Case 1: $\alpha<\beta$.

Step I. We prove that segment $\widehat{s}_{1}(\cdot)$ 's losses are more than those of segment $s_{1}(\cdot)$. Furthermore, we show that the volume of segment $\widehat{s}_{1}(\cdot)$ is less than or equal to that of segment $s_{1}(\cdot)$. The losses for segment $\widehat{s}_{1}(\cdot)$ are,

$$
\begin{aligned}
\mathcal{E}_{\widehat{s}_{1}}(\mu, \beta) & =b_{m_{2}}^{r}\left(t_{1}\right)+\widehat{x}_{1}\left(\mu_{0}-\mu\right)-\beta \\
& =b_{m}^{r}\left(t_{1}\right)+x_{1}\left(\mu_{0}-\mu\right)+x_{2}\left(\mu_{0}-\mu\right)-\alpha^{+}-\beta \\
& >b_{m}^{r}\left(t_{1}\right)+x_{1}\left(\mu_{0}-\mu\right)-\beta+x_{2}\left(\mu_{0}-\mu\right)-\beta=\mathcal{E}_{s_{1}}(\mu, \beta),
\end{aligned}
$$

where the second equality is true because of (3.20) and (3.22), the first inequality because $\alpha^{+}<\beta$ (since $\alpha<\beta$ ), and the last equality because $b_{m}^{r}\left(t_{2}\right)=0$.

Since by (3.20), $\mu_{0} \widehat{x}_{1} \leqslant \mu_{0}\left(x_{1}+x_{2}\right)$, the volume of segment $s_{1}(\cdot)$ is at least as much as the volume of segment $\widehat{s}_{1}(\cdot)$.

Step II. We prove that the occupancy of the real buffer at the end of the two segments $s_{1}(\cdot)$ and $\widehat{s}_{1}(\cdot)$ is the same.

We note that,

$$
\widehat{x}_{1}^{\prime}=x_{1}^{\prime}+x_{2}^{\prime}-\frac{\alpha^{+}}{\mu} \geqslant x_{2}^{\prime} \geqslant \frac{\beta}{\mu},
$$

where the first equality is true because of (3.20) and the first inequality because $x_{1}^{\prime}-\alpha^{+} / \mu \geqslant 0$ (from (3.19)). The inequality $\widehat{x}_{1}^{\prime} \geqslant \beta / \mu$ implies that $b_{m_{2}}^{r}\left(\hat{t}_{2}\right)=0$. Since $b_{m}^{r}\left(t_{3}\right)=0$ we conclude that $b_{m_{2}}^{r}\left(\hat{t}_{2}\right)=b_{m}^{r}\left(t_{3}\right)$.

Step III. We show that the occupancy of the fictitious buffer at the end of the two segments $s_{1}(\cdot)$ and $\widehat{s}_{1}(\cdot)$ is the same.

Indeed,

$$
\begin{aligned}
b_{m_{2}}^{f}\left(\hat{t}_{2}\right) & =b_{m_{2}}^{f}\left(t_{1}\right)+\widehat{x}_{1}\left(\mu_{0}-\mu\right)-\widehat{x}_{1}^{\prime} \mu \\
& =b_{m}^{f}\left(t_{1}\right)+x_{1}\left(\mu_{0}-\mu\right)-x_{1}^{\prime} \mu+x_{2}\left(\mu_{0}-\mu\right)-x_{2}^{\prime} \mu-\alpha^{+}+\alpha^{+}=b_{m}^{f}\left(t_{3}\right),
\end{aligned}
$$

where the second equality is true because of (3.22) and (3.20).

Step IV. We prove that $m_{2}(\cdot)$ satisfies the burstiness constraint at rate $\mu$. Note that,

$$
\begin{aligned}
b_{m_{2}}^{f}\left(\hat{t}_{1}^{\prime}\right) & =b_{m_{2}}^{f}\left(t_{1}\right)+\widehat{x}_{1}\left(\mu_{0}-\mu\right)=b_{m}^{f}\left(t_{1}\right)+\left(x_{1}+x_{2}\right)\left(\mu_{0}-\mu\right)-\alpha^{+} \\
& \leqslant b_{m}^{f}\left(t_{1}\right)+\left(x_{1}+x_{2}\right)\left(\mu_{0}-\mu\right)-\alpha=b(\mu),
\end{aligned}
$$

where the second equality is true because of (3.22) and (3.20), the first inequality because $\alpha^{+} \geqslant \alpha$ and the last equality because of (3.18). Therefore $b_{m_{2}}^{f}\left(\hat{t}_{1}^{\prime}\right) \leqslant b(\mu)$ and this inequality together with $b_{m_{2}}^{f}\left(\hat{t}_{2}\right)=b_{m}^{f}\left(t_{3}\right)$ prove that $m_{2}(\cdot)$ satisfies the burstiness constraint at the rate $\mu$.

Step V. We show that the duration of the segment $\widehat{s}_{1}(\cdot)$ is less than or equal of the duration of $s_{1}(\cdot)$. We have,

$$
\begin{aligned}
\hat{t}_{2} & =t_{1}+\widehat{x}_{1}+\widehat{x}_{1}^{\prime}=t_{1}+x_{1}+x_{2}+x_{1}^{\prime}+x_{2}^{\prime}-\frac{\alpha^{+}}{\mu_{0}-\mu}-\frac{\alpha^{+}}{\mu} \\
& =t_{3}-\frac{\alpha^{+} \mu_{0}}{\mu\left(\mu_{0}-\mu\right)} \leqslant t_{3},
\end{aligned}
$$


where the second equality is true because of (3.20) and the last inequality because $\alpha^{+} \geqslant 0$ and $\mu_{0} \geqslant \mu$.

Case 2: $\alpha \geqslant \beta$.

If $\alpha \geqslant \beta$ then $\widehat{x}_{1}^{\prime} \geqslant \beta / \mu$ because of (3.21) and therefore $b_{m_{2}}^{r}\left(\hat{t}_{2}\right)=0$.

Step I. We prove that the segments $s_{1}(\cdot)$ and $\widehat{s}_{1}(\cdot)$ have the same losses and the same volume.

The number of cells lost from segment $\widehat{s}_{1}(\cdot)$ is,

$$
\begin{aligned}
\mathcal{E}_{\widehat{s}_{1}}(\mu, \beta) & =b_{m_{2}}^{r}\left(t_{1}\right)+\widehat{x}_{1}\left(\mu_{0}-\mu\right)-\beta+b_{m_{2}}^{r}\left(\hat{t}_{2}\right)+\widehat{x}_{2}\left(\mu_{0}-\mu\right)-\beta \\
& =b_{m}^{r}\left(t_{1}\right)+x_{1}\left(\mu_{0}-\mu\right)-\beta+b_{m}^{r}\left(t_{2}\right)+x_{2}\left(\mu_{0}-\mu\right)-\beta-\alpha+\alpha \\
& =\mathcal{E}_{s_{1}}(\mu, \beta),
\end{aligned}
$$

where the second equality is true because of Eq. (3.21), (3.22) and the fact that $b_{m}^{r}\left(t_{2}\right)=0$. Since by (3.21),

$$
\mu_{0}\left(\widehat{x}_{1}+\widehat{x}_{2}\right)=\mu_{0}\left(x_{1}+x_{2}-\frac{\alpha}{\mu_{0}-\mu}+\frac{\alpha}{\mu_{0}-\mu}\right),
$$

segments $\widehat{s}_{1}(\cdot)$ and $s_{1}(\cdot)$ have the same volume.

Step II. We show that the occupancy of the real buffer at the end of the two segments $s_{1}(\cdot)$ and $\widehat{s}_{1}(\cdot)$ is the same.

We have

$$
\widehat{x}_{2}^{\prime}=x_{1}^{\prime}+x_{2}^{\prime}-\frac{\alpha}{\mu} \geqslant x_{2}^{\prime} \geqslant \frac{\beta}{\mu},
$$

where the first inequality is true because of (3.19) and the second is true by assumption. The inequality $\widehat{x}_{2}^{\prime} \geqslant \beta / \mu$ implies that $b_{m_{2}}^{r}\left(\hat{t}_{3}\right)=0$. Since $b_{m}^{r}\left(t_{3}\right)$ is also zero, we conclude that $b_{m_{2}}^{r}\left(\hat{t}_{3}\right)=b_{m}^{r}\left(t_{3}\right)$.

Step III. We prove that the occupancy of the fictitious buffer at the end of the two segments $s_{1}(\cdot)$ and $\widehat{s}_{1}(\cdot)$ is the same.

Indeed,

$$
\begin{aligned}
b_{m_{2}}^{f}\left(\hat{t}_{3}\right) & =b_{m_{2}}^{f}\left(t_{1}\right)+\widehat{x}_{1}\left(\mu_{0}-\mu\right)-\widehat{x}_{1}^{\prime} \mu+\widehat{x}_{2}\left(\mu_{0}-\mu\right)-\widehat{x}_{2}^{\prime} \mu \\
& =b_{m}^{f}\left(t_{1}\right)+x_{1}\left(\mu_{0}-\mu\right)+x_{2}\left(\mu_{0}-\mu\right)-\alpha-\alpha+\alpha-x_{1}^{\prime} \mu-x_{2}^{\prime} \mu+\alpha \\
& =b_{m}^{f}\left(t_{3}\right),
\end{aligned}
$$

where the second equality is true because of (3.21) and (3.22).

Step IV. We show that $b_{m_{2}}^{f}\left(\hat{t}_{1}^{\prime}\right)=b_{m_{2}}^{f}\left(\hat{t}_{2}^{\prime}\right)=b(\mu)$.

From the construction of the segment $m_{2}(\cdot)$ it follows that

$$
\begin{aligned}
b_{m_{2}}^{f}\left(\hat{t}_{1}^{\prime}\right) & =b_{m_{2}}^{f}\left(t_{1}\right)+\widehat{x}_{1}\left(\mu_{0}-\mu\right) \\
& =b_{m}^{f}\left(t_{1}\right)+\left(x_{1}+x_{2}\right)\left(\mu_{0}-\mu\right)-\alpha=b(\mu),
\end{aligned}
$$

and

$$
\begin{aligned}
b_{m_{2}}^{f}\left(\hat{t}_{2}^{\prime}\right) & =b_{m_{2}}^{f}\left(t_{1}\right)+\widehat{x}_{1}\left(\mu_{0}-\mu\right)-\widehat{x}_{1}^{\prime} \mu+\widehat{x}_{2}\left(\mu_{0}-\mu\right) \\
& =b_{m}^{f}\left(t_{1}\right)+\left(x_{1}+x_{2}\right)\left(\mu_{0}-\mu\right)-\alpha-\alpha+\alpha=b(\mu),
\end{aligned}
$$

where the second equality in (3.23) and (3.24) is true because of (3.21) and (3.22), and the last equality in both (3.23) and (3.24) is true because of (3.18). So $b_{m_{2}}^{f}\left(\hat{t}_{1}^{\prime}\right)=b_{m_{2}}^{f}\left(\hat{t}_{2}^{\prime}\right)=b(\mu)$, and therefore $\widehat{s}_{1}(\cdot)$ satisfies the burstiness constraint at rate $\mu$. 
Step V. We prove that the duration of the segment $\widehat{s}_{1}(\cdot)$ is equal to that of the segment $s_{1}(\cdot)$. Note that,

$$
\begin{aligned}
\hat{t}_{3} & =t_{1}+\widehat{x}_{1}+\widehat{x}_{1}^{\prime}+\widehat{x}_{2}+\widehat{x}_{2}^{\prime} \\
& =t_{1}+x_{1}+x_{2}+x_{1}^{\prime}+x_{2}^{\prime}-\frac{\alpha}{\mu_{0}-\mu}+\frac{\alpha}{\mu}+\frac{\alpha}{\mu_{0}-\mu}-\frac{\alpha}{\mu}=t_{3},
\end{aligned}
$$

where the second equality is true because of (3.21).

Consequently we can replace any segment $s_{1}(\cdot)$ of $m(\cdot)$ that consists of two consecutive ON-OFF intervals having $X_{\mathrm{OFF}} \geqslant \beta / \mu$, with the corresponding segment $\widehat{s}_{1}(\cdot)$ and the resulting message $m_{2}(\cdot)$ will have the properties described by Lemma 3.2. This completes the proof of Lemma 3.2.

Lemma 3.3. We can replace any segment $s_{2}(\cdot)$ of $m(\cdot)$, that consists of two consecutive ON-intervals that are spaced by $\widehat{X}_{\mathrm{OFF}}>b(\mu) / \mu$, with another segment of two consecutive ON-intervals with the same duration each, but separated by $\widehat{X}_{\mathrm{OFF}}=b(\mu) / \mu$. The resulting message $m_{3}(\cdot)$ satisfies the burstiness constraint at the rate $\mu$, has the same losses and percentage of loss as $m(\cdot)$, and has a smaller duration than $m(\cdot)$.

Discussion. The intuition behind this lemma is that any OFF periods that have duration greater than the maximum time $b(\mu) / \mu$ needed to empty the fictitious buffer, can be shortened to $b(\mu) / \mu$ without affecting the cell losses or the percentage of cell loss.

Proof. Consider the segment $s_{2}(\cdot)$ as shown in Fig. 10a and based on this construct the segment $\widehat{s}_{2}(\cdot)$ as shown in Fig. 10b. We assume that $x_{1}^{\prime}>b(\mu) / \mu$ and $\widehat{x}_{1}^{\prime}=b(\mu) / \mu$.

An immediate consequence of the above assumptions is that:

$$
b_{m_{3}}^{f}\left(\hat{t}_{2}\right)=b_{m}^{f}\left(t_{2}\right)=b_{m_{3}}^{r}\left(\hat{t}_{2}\right)=b_{m}^{r}\left(t_{2}\right)=0 .
$$

Therefore the new segment $\widehat{s}_{2}(\cdot)$ has the same cell loss and results in the same final buffer occupancy as the original segment $s_{2}(\cdot)$. Also they both have the same volume $\mu_{0}\left(x_{1}+x_{2}\right)$. Thus, replacing any 'type- $s_{2}(\cdot)$ ' segment by a 'type- $\widehat{s}_{2}(\cdot)$ ', results in a new message $m_{3}(\cdot)$ that has the properties described by Lemma 3.3. This completes the proof of Lemma 3.3 .

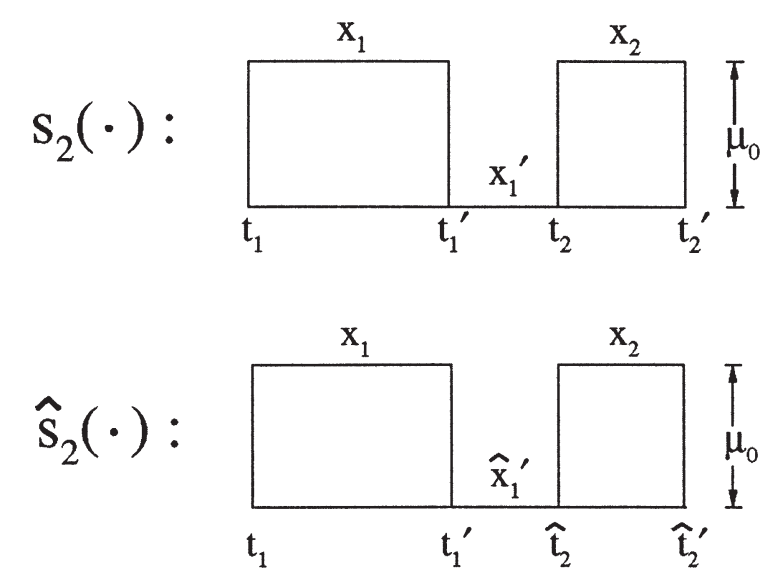

Fig. 10. Original (a) and modified (b) message segment. 


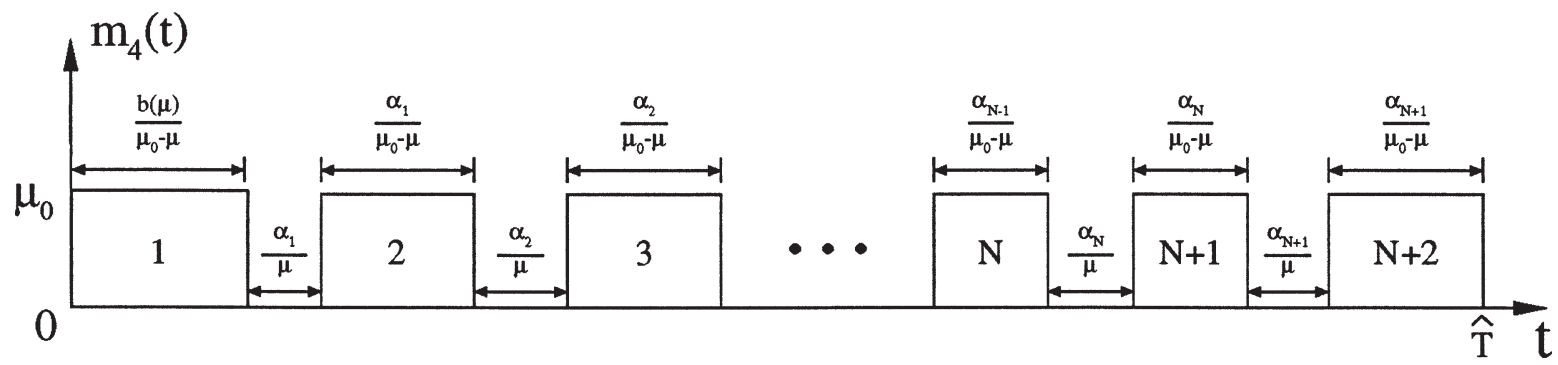

Fig. 11. Resulting message after repeated application of Lemmas 3.1-3.3.

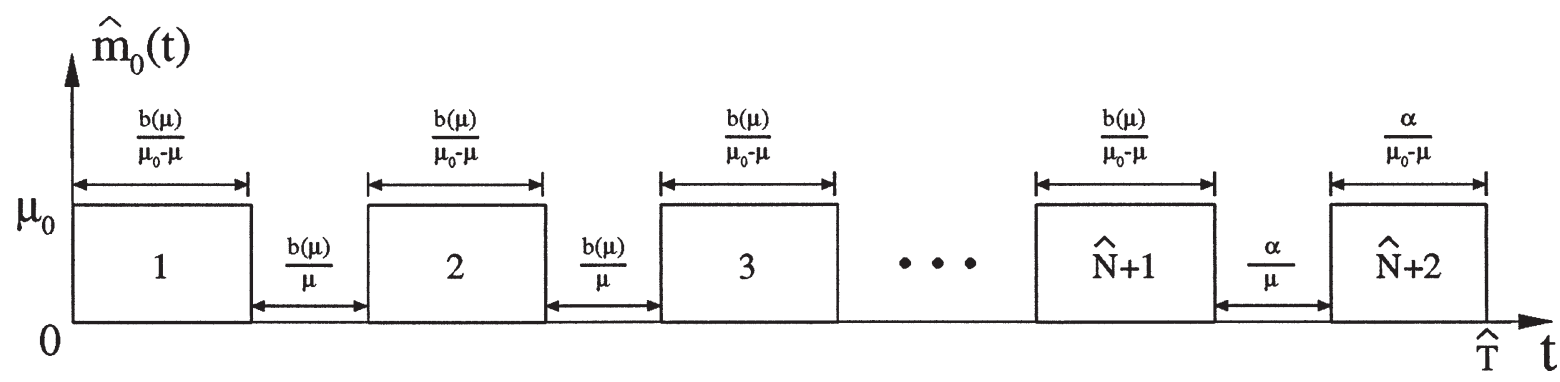

Fig. 12. Maximum cell loss message.

Repeated application of Lemmas 3.1-3.3, prove that for any $m(\cdot) \in M\left\{\mu_{0}, b(\cdot)\right\}$ that has strictly positive losses we can find an ON-OFF message $m_{4}(\cdot)$ such that: (i) $m_{4}(\cdot)$ has the form shown in Fig. 11, where $b_{m_{4}}^{f}(0)=b_{m_{4}}^{r}(0)=0, \beta \leqslant \alpha_{i} \leqslant b(\mu), 1 \leqslant i \leqslant N, 0 \leqslant \alpha_{N+1}<b(\mu)$; (ii) $m_{4}(\cdot)$ satisfies the burstiness constraint at rate $\mu$; (iii) $m_{4}(\cdot)$ has a greater or equal percentage of loss than $m(\cdot)$; and (iv) $m_{4}(\cdot)$ has equal or more losses than the original message $m(\cdot)$.

Lemma 3.4. Consider a message $m_{4}(\cdot)$ that results from repeated application of Lemmas 3.1-3.3 (see Fig. 11), and the message $\widehat{m}_{0}(\cdot)$ shown in Fig. 12. Let these messages be inputs to the single node system of Fig. 3. Assume that $b_{\widehat{m}_{0}}^{r}(0)=0$ and $0 \leqslant \alpha<b(\mu)$. Then, $\widehat{m}_{0}(\cdot)$ satisfies the burstiness constraint at the rate $\mu$, it has equal or greater percentage of cell loss than $m_{4}(\cdot)$ and incurs equal or more losses than $m_{4}(\cdot)$.

Discussion. This lemma shows that the cell loss and the percentage of cell loss are maximized when we continuously empty and then immediately refill the fictitious buffer at the maximum rate.

Proof. By construction, the content of the fictitious buffer at the end of every ON-interval, for both messages, is equal to $b(\mu)$. Therefore they both satisfy the burstiness constraint at the rate $\mu$.

We will now show that message $\widehat{m}_{0}(\cdot)$ incurs more or equal losses than $m_{4}(\cdot)$.

Equating the length of the two messages $m_{4}(\cdot)$ and $\widehat{m}_{0}(\cdot)$, we get:

$$
\begin{gathered}
\left(\sum_{i=1}^{N} \alpha_{i}+\alpha_{N+1}\right) \frac{\mu_{0}}{\mu\left(\mu_{0}-\mu\right)}=(\widehat{N} b(\mu)+\alpha) \frac{\mu_{0}}{\mu\left(\mu_{0}-\mu\right)} \\
\Leftrightarrow \sum_{i=1}^{N} \alpha_{i}+\alpha_{N+1}=\widehat{N} b(\mu)+\alpha .
\end{gathered}
$$


We also have,

$$
\begin{aligned}
\mathcal{E}_{m_{4}}(\mu, \beta) & =b(\mu)-\beta+\sum_{i=1}^{N}\left(\alpha_{i}-\beta\right)+\left(\alpha_{N+1}-\beta\right)^{+} \\
& =b(\mu)-\beta+\widehat{N} b(\mu)-N \beta+\alpha-\alpha_{N+1}+\left(\alpha_{N+1}-\beta\right)^{+},
\end{aligned}
$$

where the second equality is true because of (3.25). Furthermore,

$$
\mathcal{E}_{\widehat{m}_{0}}(\mu, \beta)=b(\mu)-\beta+\widehat{N}(b(\mu)-\beta)+(\alpha-\beta)^{+} .
$$

From (3.26) and (3.27) we get,

$$
\mathcal{E}_{\widehat{m}_{0}}(\mu, \beta)-\mathcal{E}_{m_{4}}(\mu, \beta)=(N-\widehat{N}) \beta+\alpha_{N+1}-\alpha+(\alpha-\beta)^{+}-\left(\alpha_{N+1}-\beta\right)^{+} .
$$

Since $\alpha_{i} \leqslant b(\mu), 1 \leqslant i \leqslant N$, it follows that $N \geqslant \widehat{N}$.

We consider next the following two cases: $N=\widehat{N}$ and $N>\widehat{N}$.

Case 1: $N=\widehat{N}$.

Since $\alpha_{i} \leqslant b(\mu), 1 \leqslant i \leqslant N$, and $N=\widehat{N}$, it follows that

$$
\frac{b(\mu)}{\mu_{0}-\mu}+\sum_{i=1}^{N}\left(\frac{\alpha_{i}}{\mu}+\frac{\alpha_{i}}{\mu_{0}-\mu}\right) \leqslant \frac{b(\mu)}{\mu_{0}-\mu}+\sum_{i=1}^{\widehat{N}}\left(\frac{b(\mu)}{\mu}+\frac{b(\mu)}{\mu_{0}-\mu}\right)
$$

or equivalently that,

$$
\widehat{T}-\left(\frac{\alpha_{N+1}}{\mu}+\frac{\alpha_{N+1}}{\mu_{0}-\mu}\right) \leqslant \widehat{T}-\left(\frac{\alpha}{\mu}+\frac{\alpha}{\mu_{0}-\mu}\right),
$$

which implies that $\alpha_{N+1} \geqslant \alpha$. We show that for all possible situations, $\mathcal{E}_{\widehat{m}_{0}}(\mu, \beta) \geqslant \mathcal{E}_{m_{4}}(\mu, \beta)$.

(i) $\alpha_{N+1} \geqslant \alpha>\beta$,

$$
\mathcal{E}_{\widehat{m}_{0}}(\mu, \beta)-\mathcal{E}_{m_{4}}(\mu, \beta)=\alpha_{N+1}-\alpha+\alpha-\beta-\alpha_{N+1}+\beta=0,
$$

(ii) $\alpha_{N+1}>\beta \geqslant \alpha$,

$$
\mathcal{E}_{\widehat{m}_{0}}(\mu, \beta)-\mathcal{E}_{m_{4}}(\mu, \beta)=\alpha_{N+1}-\alpha-\alpha_{N+1}+\beta=\beta-\alpha \geqslant 0,
$$

(iii) $\beta \geqslant \alpha_{N+1} \geqslant \alpha$,

$$
\mathcal{E}_{\widehat{m}_{0}}(\mu, \beta)-\mathcal{E}_{m_{4}}(\mu, \beta)=\alpha_{N+1}-\alpha \geqslant 0 .
$$

Case 2: $N \geqslant \widehat{N}+1$.

Using the above inequality in (3.28) we get,

$$
\mathcal{E}_{\widehat{m}_{0}}(\mu, \beta)-\mathcal{E}_{m_{4}}(\mu, \beta) \geqslant \beta+\alpha_{N+1}-\alpha+(\alpha-\beta)^{+}-\left(\alpha_{N+1}-\beta\right)^{+} .
$$

We consider all the possible cases and show that for each one; $\mathcal{E}_{\widehat{m}_{0}}(\mu, \beta) \geqslant \mathcal{E}_{m_{4}}(\mu, \beta)$.

(i) $\alpha>\beta$ and $\alpha_{N+1}>\beta$,

$$
\mathcal{E}_{\widehat{m}_{0}}(\mu, \beta)-\mathcal{E}_{m_{4}}(\mu, \beta)=\beta+\alpha_{N+1}-\alpha+\alpha-\beta-\alpha_{N+1}+\beta=\beta>0,
$$

(ii) $\alpha_{N+1}>\beta \geqslant \alpha$,

$$
\mathcal{E}_{\widehat{m}_{0}}(\mu, \beta)-\mathcal{E}_{m_{4}}(\mu, \beta)=\beta+\alpha_{N+1}-\alpha-\alpha_{N+1}+\beta=\beta-\alpha+\beta>0,
$$


(iii) $\alpha>\beta \geqslant \alpha_{N+1}$,

$$
\mathcal{E}_{\widehat{m}_{0}}(\mu, \beta)-\mathcal{E}_{m_{4}}(\mu, \beta)=\beta+\alpha_{N+1}-\alpha+\alpha-\beta=\alpha_{N+1} \geqslant 0,
$$

(iv) $\beta \geqslant \alpha_{N+1}$ and $\beta \geqslant \alpha$,

$$
\mathcal{E}_{\widehat{m}_{0}}(\mu, \beta)-\mathcal{E}_{m_{4}}(\mu, \beta)=\alpha_{N+1}+\beta-\alpha>0 .
$$

What remains to be shown is that message $\widehat{m}_{0}(\cdot)$ has a greater or equal percentage of cell loss than $m_{4}(\cdot)$. The volume for message $m_{4}(\cdot)$ is,

$$
\frac{\mu_{0}}{\mu_{0}-\mu} b(\mu)+\frac{\mu_{0}}{\mu_{0}-\mu}\left(\sum_{i=1}^{N} \alpha_{i}+\alpha_{N+1}\right)
$$

whereas the volume for message $\widehat{m}_{0}(\cdot)$ is,

$$
\frac{\mu_{0}}{\mu_{0}-\mu} b(\mu)+\frac{\mu_{0}}{\mu_{0}-\mu}(\widehat{N} b(\mu)+\alpha)
$$

Equation (3.25) establishes the fact that the two volumes are equal. Since there are equal or more losses for message $\widehat{m}_{0}(\cdot)$ than for $m_{4}(\cdot)$, it is clear that the percentage of loss for $\widehat{m}_{0}(\cdot)$ is at least as much as for $m_{4}(\cdot)$.

The proof of Lemma 3.4 is now complete.

Proof of Theorem 3.1. The proof of Theorem 3.1 follows directly from Lemmas 3.1-3.4.

The upper bound on the percentage of cell loss in the first node among all $m(\cdot)$ in $M\left\{\mu_{0}, b(\cdot)\right\}$, that is the percentage of cell loss for the message $\widehat{m}_{0}(\cdot)$, is given by the following equation:

$$
\begin{aligned}
\mathcal{L}_{\widehat{m}_{0}}(\mu, \beta) & =\frac{(\widehat{N}+1)(b(\mu)-\beta)+(\alpha-\beta)^{+}}{(\widehat{N}+1) b(\mu) \mu_{0} /\left(\mu_{0}-\mu\right)+\alpha \mu_{0} /\left(\mu_{0}-\mu\right)} \\
& =\frac{\mu_{0}-\mu}{\mu_{0}} \cdot \frac{(\widehat{N}+1)(b(\mu)-\beta)+(\alpha-\beta)^{+}}{(\widehat{N}+1) b(\mu)+\alpha},
\end{aligned}
$$

where the first equality is true because of (3.27) and (3.29).

Because Theorem 3.1 guarantees that the message $\widehat{m}_{0}(\cdot)$ satisfies the burstiness constraint only at the rate $\mu$, this upper bound may not be tight.

From (3.30) it is clear that when $\alpha \leqslant \beta$,

$$
\mathcal{L}_{\widehat{m}_{0}}(\mu, \beta) \leqslant \frac{\mu_{0}-\mu}{\mu_{0}} \cdot \frac{b(\mu)-\beta}{b(\mu)} .
$$

In the case where $\alpha>\beta$ we have,

$$
\frac{(\widehat{N}+1)(b(\mu)-\beta)+\alpha-\beta}{(\widehat{N}+1) b(\mu)+\alpha} \leqslant \frac{(\widehat{N}+1)(b(\mu)-\beta)+\alpha-\beta+b(\mu)-\alpha}{(\widehat{N}+1) b(\mu)+\alpha+b(\mu)-\alpha} .
$$

The above inequality is true because $b(\mu)-\alpha>0$, and the fraction on the left side is not greater than 1 . Therefore since $\mu_{0}>\mu$, inequality (3.31) is also true when $\alpha>\beta$.

We now proceed in the formulation and solution of Problem (P2). 
3.1.2. The problem in the remaining nodes: formulation and solution of $(P 2)$

Consider the work conserving system of Fig. 13 that has a finite buffer $\beta$ and a maximum service rate $\mu$.

We consider input messages $m(\cdot)$ that have a finite duration $T$ and maximum input rate $\mu_{0}$; that is $m(\cdot) \in$ $M^{\prime}\left\{\mu_{0}\right\}$ where,

$$
M^{\prime}\left\{\mu_{0}\right\} \triangleq\left\{m(\cdot) ; m(t) \leqslant \mu_{0}, 0 \leqslant t \leqslant T\right\}
$$

We make the same assumptions as in Section 3.1.1. That is we assume that $\mu_{0}>\mu$, and that the buffer occupancy at the beginning of the transmission session is zero. We also denote by $\mathcal{L}_{m}(\mu, \beta)$ the percentage of cell loss and by $\mathcal{E}_{m}(\mu, \beta)$ the number of cells lost for message $m(\cdot)$ as it passes through the single node system. The problem that we want to solve is the following:

(P2) Determine an input message $\widehat{m}_{0}^{\prime}(\cdot) \in M^{\prime}\left\{\mu_{0}\right\}$ such that,

$$
\mathcal{L}_{m}(\mu, \beta) \leqslant \mathcal{L}_{\widehat{m}_{0}^{\prime}}(\mu, \beta), \quad \forall m(\cdot) \in M^{\prime}\left\{\mu_{0}\right\}
$$

The main result of this section is summarized by the following theorem:

Theorem 3.2. The message $\widehat{m}_{0}^{\prime}(\cdot)$ that belongs to $M^{\prime}\left\{\mu_{0}\right\}$ and suffers the maximum percentage of loss when passing through the single node system of Fig. 13, is the one that inputs cells with the maximum input rate $\mu_{0}$. That message also incurs the maximum number of losses.

Proof of Theorem 3.2. Let $m(\cdot)$ be any message in $M^{\prime}\left\{\mu_{0}\right\}$ with strictly positive losses. We can then use (3.12) to represent the number of cells dropped as a result of buffer overflow during the transmission of $m(\cdot)$. That is,

$$
\mathcal{E}_{m}(\mu, \beta)=\sum_{i=1}^{N}\left(\int_{\tau_{i}}^{s_{i}}(m(r)-\mu) \mathrm{d} r-\beta\right),
$$

where $N,\left\{\tau_{i}, s_{i}\right\}_{i=1}^{N}$ are defined as in (3.12). Let $A \triangleq \bigcup_{i=1}^{N}\left[\tau_{i}, s_{i}\right]$ and define $\widehat{m}_{0}^{\prime}(\cdot)$ as follows,

$$
\widehat{m}_{0}^{\prime}(t) \triangleq \begin{cases}\mu_{0} & \text { if } 0 \leqslant t \leqslant T \\ 0 & \text { otherwise. }\end{cases}
$$

The following then is true,

$$
\begin{aligned}
& \mathcal{E}_{m}(\mu, \beta)=\int_{A}(m(r)-\mu) \mathrm{d} r-N \beta \leqslant \int_{A}\left(\mu_{0}-\mu\right) \mathrm{d} r-N \beta \\
& =\left(\mu_{0}-\mu\right) \sum_{i=1}^{N}\left(s_{i}-\tau_{i}\right)-N \beta \leqslant\left(\mu_{0}-\mu\right) T-N \beta \\
& \leqslant\left(\mu_{0}-\mu\right) T-\beta=\mathcal{E}_{\widehat{m}_{0}^{\prime}}(\mu, \beta),
\end{aligned}
$$

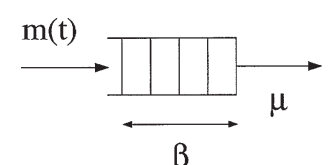

Fig. 13. Single node model 
where the first inequality is true because $m(\cdot) \leqslant \mu_{0}$, the second because $\sum_{i=1}^{N}\left(s_{i}-\tau_{i}\right) \leqslant T$ and the last one because $N \geqslant 1$.

Inequality (3.35) establishes the fact that $\widehat{m}_{0}^{\prime}(\cdot)$ incurs the maximum number of losses among all messages in $M^{\prime}\left\{\mu_{0}\right\}$. We now need to show that $\widehat{m}_{0}^{\prime}(\cdot)$ incurs also the maximum percentage of loss.

We define $\bar{A}$ to be the complement of $A$ in $[0, T]$, that is,

$$
\bar{A} \triangleq \bigcap_{i=1}^{N}\left[\tau_{i}, s_{i}\right]^{c} \cap[0, T] .
$$

The following then is true,

$$
\begin{aligned}
\mathcal{L}_{m}(\mu, \beta) & =\frac{\int_{A}(m(r)-\mu) \mathrm{d} r-N \beta}{\int_{A} m(r) \mathrm{d} r+\int_{\bar{A}} m(r) \mathrm{d} r} \leqslant \frac{\int_{A}(m(r)-\mu) \mathrm{d} r-N \beta}{\int_{A} m(r) \mathrm{d} r} \\
& \leqslant \frac{\int_{A}\left(\mu_{0}-m(r)\right) \mathrm{d} r+\int_{A}(m(r)-\mu) \mathrm{d} r-N \beta}{\int_{A}\left(\mu_{0}-m(r)\right) \mathrm{d} r+\int_{A} m(r) \mathrm{d} r} \\
& =\frac{\int_{A}\left(\mu_{0}-\mu\right) \mathrm{d} r-N \beta}{\int_{A} \mu_{0} \mathrm{~d} r}=1-\frac{\mu}{\mu_{0}}-\frac{N \beta}{\mu_{0} \sum_{i=1}^{N}\left(s_{i}-\tau_{i}\right)},
\end{aligned}
$$

where the first inequality is true because $\int_{\bar{A}} m(r) \mathrm{d} r \geqslant 0$, and the second because $\int_{A}\left(\mu_{0}-m(r)\right) \mathrm{d} r \geqslant 0$ and the fact that the fraction on the right part of the first inequality is less than one.

The percentage of loss for message $\widehat{m}_{0}^{\prime}(\cdot)$ is,

$$
\mathcal{L}_{\widehat{m}_{0}^{\prime}}(\mu, \beta)=\frac{\left(\mu_{0}-\mu\right) T-\beta}{\mu_{0} T}=1-\frac{\mu}{\mu_{0}}-\frac{\beta}{\mu_{0} T} .
$$

Furthermore,

$$
N \geqslant 1 \geqslant \frac{\sum_{i=1}^{N}\left(s_{i}-\tau_{i}\right)}{T} .
$$

From (3.36), (3.37) and (3.38) we obtain,

$$
\mathcal{L}_{m}(\mu, \beta) \leqslant \mathcal{L}_{\widehat{m}_{0}^{\prime}}(\mu, \beta), \quad \forall m(\cdot) \in M^{\prime}\left\{\mu_{0}\right\}
$$

and this completes the proof of Theorem 3.2.

In the next section we combine the results from problems (P1) and (P2), to find an upper bound on the percentage of cell loss along the route.

\subsubsection{An expression on the maximum percentage of cell loss along the route}

Theorem 3.1 and inequality (3.31) establish the fact that,

$$
\mathcal{L}_{m_{0}}^{1}(\boldsymbol{\mu}, \boldsymbol{\beta}) \leqslant \frac{\mu_{0}-\mu_{1}}{\mu_{0}} \cdot \frac{b\left(\mu_{1}\right)-\beta_{1}}{b\left(\mu_{1}\right)}, \quad \forall m_{0}(\cdot) \in M\left\{\mu_{0}, b(\cdot)\right\} .
$$

From Theorem 3.2 and Eq. (3.37) we have that,

$$
\mathcal{L}_{m_{0}}^{i}(\boldsymbol{\mu}, \boldsymbol{\beta}) \leqslant 1-\frac{\mu_{i}}{\mu_{i-1}}-\frac{\beta_{i}}{\mu_{i-1} \widehat{T}_{i-1}}, \quad \forall m_{0}(\cdot) \in M\left\{\mu_{0}, b(\cdot)\right\}, \quad 2 \leqslant i \leqslant P,
$$


where $\widehat{T}_{i}$ is the upper bound on the duration of message $m_{i}(\cdot)$, and is given by Eq. (3.2).

From (3.39), (3.40) and (3.3) we conclude that,

$$
\mathcal{L}_{m_{0}}(\boldsymbol{\mu}, \boldsymbol{\beta}) \leqslant \widehat{\mathcal{L}}(\boldsymbol{\mu}, \boldsymbol{\beta}), \quad \forall m_{0}(\cdot) \in M\left\{\mu_{0}, b(\cdot)\right\},
$$

where,

$$
\widehat{\mathcal{L}}(\boldsymbol{\mu}, \boldsymbol{\beta})=1-\left(\frac{\mu_{1}}{\mu_{0}}+\frac{\beta_{1}\left(\mu_{0}-\mu_{1}\right)}{\mu_{0} b\left(\mu_{1}\right)}\right) \prod_{i=2}^{P}\left(\frac{\mu_{i}}{\mu_{i-1}}+\frac{\beta_{i}}{\mu_{i-1}\left(\widehat{T}+\sum_{j=1}^{i-1} \beta_{j} / \mu_{j}\right)}\right) .
$$

Equation (3.42) provides, for a fixed allocation, an upper bound on the percentage of cell loss along a fixed route. Even though this upper bound is not tight, it is intuitively pleasing as it captures the interaction between resource allocation and percentage of cell loss along a fixed route.

The upper bound on the end-to-end cell delay along a fixed route is discussed in the next section.

\subsection{Upper bound on the end-to-end cell delay along a fixed route}

Consider the multinode system of Fig. 14, where $d_{i}$ is the sum of the processing time in node $i$ and the propagation delay from node $i$ to node $i+1$. Node $P+1$ refers to the destination. We assume that the input message $m_{0}(\cdot)$ belongs to the family $M\left\{\mu_{0}, b(\cdot)\right\}$.

For the same reason as in Section 3.1 we restrict attention to allocations that satisfy:

$$
\begin{aligned}
& \mu_{k} \leqslant \mu_{k-1}, \quad 1 \leqslant k \leqslant P, \\
& \beta_{1} \leqslant \min \left(b\left(\mu_{1}\right), \widehat{T}\left(\mu_{0}-\mu_{1}\right)\right), \\
& \beta_{k} \leqslant \widehat{T}_{k-1}\left(\mu_{k-1}-\mu_{k}\right), \quad 2 \leqslant k \leqslant P,
\end{aligned}
$$

where,

$$
\widehat{T}_{k}=\widehat{T}+\sum_{i=1}^{k} \frac{\beta_{k}}{\mu_{k}}, \quad 1 \leqslant k \leqslant P-1 .
$$

The problem that we want to solve in this section is the following:

For a fixed resource allocation $(\boldsymbol{\mu}, \boldsymbol{\beta})=\left\{\mu_{i}, \beta_{i}\right\}_{1 \leqslant i \leqslant P}$ that satisfies the assumptions given by (3.43)-(3.46), find a function $\widehat{\mathcal{D}}(\boldsymbol{\mu}, \boldsymbol{\beta})$ such that:

$$
\mathcal{D}_{m_{0}}(\boldsymbol{\mu}, \boldsymbol{\beta}) \leqslant \widehat{\mathcal{D}}(\boldsymbol{\mu}, \boldsymbol{\beta}), \quad \forall m_{0}(\cdot) \in M\left\{\mu_{0}, b(\cdot)\right\},
$$

where $\mathcal{D}_{m_{0}}(\boldsymbol{\mu}, \boldsymbol{\beta})$ is the maximum possible end-to-end delay over all packets in message $m_{0}(\cdot)$, as they travel through the multinode system of Fig. 14.

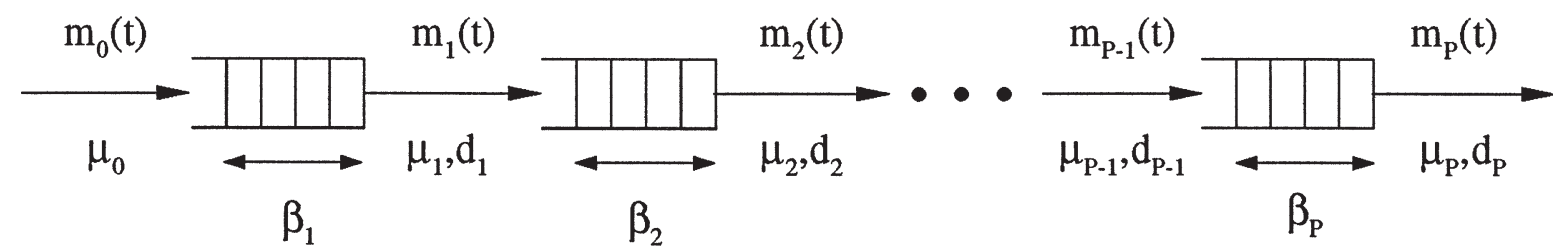

Fig. 14. Connection spanning $P$ nodes in sequence. 
The maximum end-to-end delay suffered by a cell that travels along the fixed route of Fig. 14, without being lost, can be obtained by inspection and is given by the following equation:

$$
\widehat{\mathcal{D}}(\boldsymbol{\mu}, \boldsymbol{\beta})=\sum_{k=1}^{P}\left(\frac{\beta_{k}}{\mu_{k}}+d_{k}\right) .
$$

Here we present a message that satisfies the burstiness constraint at rate $\mu_{1}$ and achieves this bound. Given that, $\beta_{1} /\left(\mu_{0}-\mu_{1}\right) \leqslant \widehat{T}$ (see (3.44)), we consider the following message $\widehat{m}_{0}(\cdot)$ :

$$
\widehat{m}_{0}(t)= \begin{cases}\mu_{0}, & \text { if } 0 \leqslant t \leqslant \frac{\beta_{1}}{\mu_{0}-\mu_{1}} \\ \mu_{1}, & \text { if } \frac{\beta_{1}}{\mu_{0}-\mu_{1}}<t \leqslant \widehat{T} .\end{cases}
$$

The burstiness of $\widehat{m}_{0}(\cdot)$ at the rate $\mu_{1}$ is given by,

$$
b_{\widehat{m}_{0}}\left(\mu_{1}\right) \triangleq \sup _{0 \leqslant s \leqslant t \leqslant \widehat{T}} \int_{s}^{t}\left(\widehat{m}_{0}(r)-\mu_{1}\right) \mathrm{d} r=\left(\mu_{0}-\mu_{1}\right) \frac{\beta_{1}}{\mu_{0}-\mu_{1}}=\beta_{1} \leqslant b\left(\mu_{1}\right),
$$

and this shows that $\widehat{m}_{0}(\cdot)$ satisfies the burstiness constraint at rate $\mu_{1}$.

Consider next the last packet of $\widehat{m}_{0}(\cdot)$, i.e., the packet that arrives to the first node at time $\widehat{T}$. We will show that this packet may suffer the maximum possible delay, given by Eq. (3.47).

We assume that the last packet of $\widehat{m}_{0}(\cdot)$ is not lost due to buffer overflow and we denote by $\hat{t}_{k}, 1 \leqslant k \leqslant P$ the arrival time of that packet to the $k+1$ node. We also denote by $\widehat{\xi}_{k}(t), 1 \leqslant k \leqslant P$ the buffer occupancy of buffer $k$ at time $t$ and by $\widehat{m}_{k}(\cdot), 1 \leqslant k \leqslant P$ the input message at the $k+1$ node.

Based on the above, the following is true:

At the first node:

$$
\begin{aligned}
& \widehat{\xi}_{1}(\widehat{T})=\min \left(\left(\mu_{0}-\mu_{1}\right) \beta_{1} /\left(\mu_{0}-\mu_{1}\right), \beta_{1}\right)=\beta_{1}, \\
& \hat{t}_{1}=\widehat{T}+\beta_{1} / \mu_{1}+d_{1}, \\
& \widehat{m}_{1}(t)=\mu_{1}, \quad d_{1} \leqslant t \leqslant \hat{t}_{1} .
\end{aligned}
$$

At the subsequent nodes: $2 \leqslant k \leqslant P$

$$
\begin{aligned}
& \widehat{\xi}_{k}\left(\hat{t}_{k-1}\right)=\min \left(\left(\mu_{k-1}-\mu_{k}\right)\left(\hat{t}_{k-1}-\sum_{j=1}^{k-1} d_{j}\right), \beta_{k}\right) \\
&=\min \left(\left(\mu_{k-1}-\mu_{k}\right)\left(\widehat{T}+\sum_{j=1}^{k-1} \beta_{j} / \mu_{j}\right), \beta_{k}\right)=\beta_{k}, \\
& \hat{t}_{k}=\hat{t}_{k-1}+\beta_{k} / \mu_{k}+d_{k}=\widehat{T}+\sum_{j=1}^{k}\left(\beta_{j} / \mu_{j}+d_{j}\right), \\
& \widehat{m}_{k}(t)=\mu_{k}, \quad \sum_{j=1}^{k} d_{j} \leqslant t \leqslant \hat{t}_{k},
\end{aligned}
$$

where we have used the assumptions made in (3.45), (3.46). 
The total end-to-end cell delay is then given by the following expression:

$$
\mathcal{D}_{\widehat{m}_{0}}(\boldsymbol{\mu}, \boldsymbol{\beta})=\hat{t}_{P}-\widehat{T}=\sum_{k=1}^{P}\left(\frac{\beta_{k}}{\mu_{k}}+d_{k}\right),
$$

which shows that $\widehat{m}_{0}$ achieves the upper bound given by (3.47).

The above discussion can be summarized as follows:

Theorem 3.3. The maximum end-to-end cell delay over all messages in $M\left\{\mu_{0}, b(\cdot)\right\}$ and over all packets on these messages, is upper bounded by the following function:

$$
\widehat{\mathcal{D}}(\boldsymbol{\mu}, \boldsymbol{\beta})=\sum_{k=1}^{P}\left(\frac{\beta_{k}}{\mu_{k}}+d_{k}\right) .
$$

There is a message that satisfies the burstiness constraint at rate $\mu_{1}$ and achieves this upper bound.

Even though the upper bound in (3.49) is not tight, it is intuitively pleasing as it captures the interaction among resource allocation and end-to-end cell delay along a fixed route.

Equations (3.49) and (3.42) conclude the solution of Step 1 as presented in Section 2. The solution of Step 2 is given in the next section.

\section{The optimization problem}

Define the set $\mathcal{N}$ of all service allocation decisions:

$$
(\boldsymbol{x}, \boldsymbol{w}, \boldsymbol{\mu}, \boldsymbol{\beta}) \triangleq\left\{x_{s}, w_{s},\left\{\mu_{i}^{s}, \beta_{i}^{s}\right\}_{i \in r_{s}}\right\}_{s \in S},
$$

that satisfy the following conditions:

$$
\begin{aligned}
& 0 \leqslant x_{s} \leqslant R_{s}\left(w_{s}\right), \quad \forall s \in S, \\
& w_{s} \geqslant 0, \quad \forall s \in S, \\
& \sum_{s \in S} x_{s} \mu_{l}^{s} \leqslant C_{l}(k \widehat{T}) \quad \text { and } \sum_{s \in S} x_{s} \beta_{l}^{s} \leqslant B_{l}(k \widehat{T}), \quad \forall l \in L, \\
& \left\{0<\mu_{i}^{s} \leqslant \mu_{i-1}^{s}\right\}_{i \in r_{s}}, \quad \forall s \in S, \\
& 0 \leqslant \beta_{1}^{s} \leqslant \min \left(b_{s}\left(\mu_{1}^{s}\right), \widehat{T}\left(\mu_{0}^{s}-\mu_{1}^{s}\right)\right), \quad \forall s \in S, \\
& 0 \leqslant \beta_{i}^{s} \leqslant\left(\mu_{i-1}^{s}-\mu_{i}^{s}\right) T_{i}^{s}, \quad \forall i>1, \forall s \in S, \\
& \widehat{\mathcal{L}}_{s}(\boldsymbol{\mu}, \boldsymbol{\beta}) \leqslant L_{s}, \quad \forall s \in S, \\
& \widehat{\mathcal{D}}_{s}(\boldsymbol{\mu}, \boldsymbol{\beta}) \leqslant D_{s}, \quad \forall s \in S,
\end{aligned}
$$

where: $R_{s}\left(w_{s}\right)=\lambda_{s} \widehat{T} \exp \left(-w_{s}\right)$ is the aggregate user demand function; $C_{l}(k \widehat{T})$ and $B_{l}(k \widehat{T})$ are the bandwidth and buffers, respectively, that are available for allocation at the beginning of the $k$ th allocation period at node $l \in L ; T_{1}^{s}=\widehat{T} ; T_{i}^{s}=T_{i-1}^{s}+\beta_{i}^{s} / \mu_{i}^{s}, i>1$; and the functions $\widehat{\mathcal{L}}_{s}(\boldsymbol{\mu}, \boldsymbol{\beta})$ and $\widehat{\mathcal{D}}_{s}(\boldsymbol{\mu}, \boldsymbol{\beta})$ are given by Eqs $(3.42)$ 
and (3.49), respectively. The constraints expressed by (4.1)-(4.3), (4.7) and (4.8) are the same as (2.4)-(2.6) and (2.12), respectively, and they have been discussed in Section 2. The constraints (4.4)-(4.6) are the same as (3.1a)-(3.1c), respectively, and have been discussed in Section 3.1.

Using the results from Section 3, we can conclude that if a service allocation decision belongs to the set $\mathcal{N}$, it is conformant. Therefore, the optimization problem of Step 2 of our approach to Service Provisioning (cf. Section 2) becomes:

Find a service allocation decision $(\overline{\boldsymbol{x}}, \overline{\boldsymbol{w}}, \overline{\boldsymbol{\mu}}, \overline{\boldsymbol{\beta}}) \in \mathcal{N}$, if such one exists, that maximizes the welfare function:

$$
W(\boldsymbol{x}, \boldsymbol{w}, \boldsymbol{\mu}, \boldsymbol{\beta})=\sum_{s \in S} x_{s} w_{s}+\sum_{s \in S} \int_{w_{s}}^{\infty} \min \left(x_{s}, R_{s}(u)\right) \mathrm{d} u
$$

The existence of a welfare maximizing solution to the optimization problem formulated above is given by Theorem 4.1 that follows. The theorem also suggests a procedure for determining a welfare maximizing solution. This procedure is informationally decentralized in nature and is similar in spirit to the iterative solution process proposed in [16].

Theorem 4.1. (i) There exists a (conformant) service allocation decision $(\overline{\boldsymbol{x}}, \overline{\boldsymbol{w}}, \overline{\boldsymbol{\mu}}, \overline{\boldsymbol{\beta}}) \in \mathcal{N}$ that is welfare maximizing. (ii) Determining a welfare maximizing allocation is equivalent to solving the following multi-person game:

$$
\begin{aligned}
& \text { Player } N \text { (Network) : } \min _{\left(\boldsymbol{\lambda}^{1}, \boldsymbol{\lambda}^{2}\right) \geqslant 0} G\left(\boldsymbol{\mu}, \boldsymbol{\beta}, \boldsymbol{\lambda}^{1}, \boldsymbol{\lambda}^{2}\right), \\
& \text { Player } \left.U_{s} \text { (Users of Service } s ; s \in S\right): \min _{\left(\boldsymbol{\mu}_{s}, \boldsymbol{\beta}_{s}\right) \in K_{s}}\left(\left\langle\boldsymbol{\lambda}^{1}, \boldsymbol{\mu}_{s}\right\rangle+\left\langle\boldsymbol{\lambda}^{2}, \boldsymbol{\beta}_{s}\right\rangle\right),
\end{aligned}
$$

where: $\left(\boldsymbol{\mu}_{s}, \boldsymbol{\beta}_{s}\right)=\left\{\mu_{i}^{s}, \beta_{i}^{s}\right\}_{i \in r_{s}} ; K_{s}$ is the set of resource allocations, for a type s service, that satisfy conditions (4.4)-(4.8); $\langle\boldsymbol{x}, \boldsymbol{y}\rangle$ is the inner product of the vectors $\boldsymbol{x}, \boldsymbol{y} ; \boldsymbol{\lambda}^{j}=\left\{\lambda_{l}^{j}\right\}_{l \in L}, j=1,2 ; \boldsymbol{C}^{\prime}=$ $\left\{C_{l}^{\prime}\right\}_{l \in L}, \boldsymbol{B}^{\prime}=\left\{B_{l}^{\prime}\right\}_{l \in L} ; G\left(\boldsymbol{\mu}, \boldsymbol{\beta}, \boldsymbol{\lambda}^{1}, \boldsymbol{\lambda}^{2}\right) \triangleq \sum_{s \in S}\left(\lambda_{s} \widehat{T} \exp \left(-\left\langle\boldsymbol{\lambda}^{1}, \boldsymbol{\mu}_{s}\right\rangle-\left\langle\boldsymbol{\lambda}^{2}, \boldsymbol{\beta}_{s}\right\rangle\right)\right)+\left\langle\boldsymbol{\lambda}^{1}, \boldsymbol{C}^{\prime}\right\rangle+\left\langle\boldsymbol{\lambda}^{2}, \boldsymbol{B}^{\prime}\right\rangle$. The solution $\left(\overline{\boldsymbol{\lambda}}^{1}, \overline{\boldsymbol{\lambda}^{2}}, \overline{\boldsymbol{\mu}}, \overline{\boldsymbol{\beta}}\right)$ of the above multiperson game determines the price $\bar{w}_{s}=\left\langle\overline{\boldsymbol{\lambda}}^{1}, \overline{\boldsymbol{\mu}}_{s}\right\rangle+\left\langle\overline{\boldsymbol{\lambda}}^{2}, \overline{\boldsymbol{\beta}}_{s}\right\rangle$ for a type service, and the corresponding offered amount $\bar{x}_{s}=\lambda_{s} \widehat{T} \exp \left(-\bar{w}_{s}\right)$.

Proof of (i). The proof of part (i) of the theorem is based on Lemmas 4.1 and 4.2 that follow. Qualitatively, the optimization problem formulated in this section is similar to the one investigated in [16]. For that reason the proofs of the two lemmas are omitted. A detailed proof of the lemmas can be found in [24].

Let $K$ be the set of resource allocations that satisfy conditions (4.4)-(4.8).

Lemma 4.1. For any fixed resource allocation $\{\boldsymbol{\mu}, \boldsymbol{\beta}\} \in K$, the maximum of the social welfare function (4.9) subject to (4.1)-(4.3) is given by

$$
W(\overline{\boldsymbol{x}}, \overline{\boldsymbol{w}}, \boldsymbol{\mu}, \boldsymbol{\beta})=\min _{\left(\boldsymbol{\lambda}^{1}, \boldsymbol{\lambda}^{2}\right) \geqslant 0} G\left(\boldsymbol{\mu}, \boldsymbol{\beta}, \boldsymbol{\lambda}^{1}, \boldsymbol{\lambda}^{2}\right),
$$

where: $G\left(\boldsymbol{\mu}, \boldsymbol{\beta}, \boldsymbol{\lambda}^{1}, \boldsymbol{\lambda}^{2}\right)$ is the function defined in the statement of Theorem $4.1 ;\left(\overline{\boldsymbol{\lambda}}^{1}, \overline{\boldsymbol{\lambda}}^{2}\right)$ is the minimizer in (4.12); $\bar{w}_{s}=\left\langle\overline{\boldsymbol{\lambda}}^{1}, \overline{\boldsymbol{\mu}}_{s}\right\rangle+\left\langle\overline{\boldsymbol{\lambda}}^{2}, \overline{\boldsymbol{\beta}}_{s}\right\rangle, \bar{x}_{s}=\lambda_{s} \widehat{T} \exp \left(-\bar{w}_{s}\right)$, for all $s \in S$.

In view of Lemma 4.1 the maximum welfare function is given by:

$$
W(\overline{\boldsymbol{x}}, \overline{\boldsymbol{w}}, \overline{\boldsymbol{\mu}}, \overline{\boldsymbol{\beta}})=\max _{(\boldsymbol{\mu}, \boldsymbol{\beta}) \in K} W(\overline{\boldsymbol{x}}, \overline{\boldsymbol{w}}, \boldsymbol{\mu}, \boldsymbol{\beta})=\max _{(\boldsymbol{\mu}, \boldsymbol{\beta}) \in K} \min _{\left(\boldsymbol{\lambda}^{1}, \boldsymbol{\lambda}^{2}\right) \geqslant 0} G\left(\boldsymbol{\mu}, \boldsymbol{\beta}, \boldsymbol{\lambda}^{1}, \boldsymbol{\lambda}^{2}\right) .
$$

Thus, determining the maximum of the welfare function is equivalent to solving the max-min problem in (4.13). 
Lemma 4.2. There exists a saddle point $\left(\overline{\boldsymbol{\mu}}, \overline{\boldsymbol{\beta}}, \overline{\boldsymbol{\lambda}}^{1}, \overline{\boldsymbol{\lambda}}^{2}\right)$ to the max-min problem in (4.13) that is welfare maximizing.

The proof of part (i) follows directly from Lemmas 4.1 and 4.2.

Proof of (ii). In view of Eq. (4.13) and the expression for $G\left(\boldsymbol{\mu}, \boldsymbol{\beta}, \boldsymbol{\lambda}^{1}, \boldsymbol{\lambda}^{2}\right)$ (given in the statement of the theorem), determining a welfare maximizing allocation is equivalent to solving the following multi-person game:

Player $N$ (Network): $\min _{\left(\boldsymbol{\lambda}^{1}, \boldsymbol{\lambda}^{2}\right) \geqslant 0} G\left(\boldsymbol{\mu}, \boldsymbol{\beta}, \boldsymbol{\lambda}^{1}, \boldsymbol{\lambda}^{2}\right)$,

Player $U_{s}$ (Users of Service $\left.s ; s \in S\right): \min _{\left(\boldsymbol{\mu}_{s}, \boldsymbol{\beta}_{s}\right) \in K_{s}}\left(\left\langle\boldsymbol{\lambda}^{1}, \boldsymbol{\mu}_{s}\right\rangle+\left\langle\boldsymbol{\lambda}^{2}, \boldsymbol{\beta}_{s}\right\rangle\right)$,

where $K_{s}$ is the set of resource allocations for a service of type $s$, that satisfy the following conditions:

$$
\begin{aligned}
& \left\{0<\mu_{i}^{s} \leqslant \mu_{i-1}^{s}\right\}_{i \in r_{s}}, \\
& 0 \leqslant \beta_{1}^{s} \leqslant \min \left(b_{s}\left(\mu_{1}^{s}\right), \widehat{T}\left(\mu_{0}^{s}-\mu_{1}^{s}\right)\right), \\
& 0 \leqslant \beta_{i}^{s} \leqslant\left(\mu_{i-1}^{s}-\mu_{i}^{s}\right) T_{i}^{s}, \quad \forall i>1, \\
& \widehat{\mathcal{L}}_{s}(\boldsymbol{\mu}, \boldsymbol{\beta}) \leqslant L_{s}, \\
& \widehat{\mathcal{D}}_{s}(\boldsymbol{\mu}, \boldsymbol{\beta}) \leqslant D_{s},
\end{aligned}
$$

where: $T_{1}^{s}=\widehat{T} ; T_{i}^{s}=T_{i-1}^{s}+\beta_{i}^{s} / \mu_{i}^{s}, i>1$; and $\widehat{\mathcal{L}}_{s}(\boldsymbol{\mu}, \boldsymbol{\beta}), \widehat{\mathcal{D}}_{s}(\boldsymbol{\mu}, \boldsymbol{\beta})$ are given by (3.42) and (3.49), respectively. The values $\lambda_{l}^{1}, \lambda_{l}^{2}$ can be interpreted as the rent, for one unit of bandwidth and buffer, respectively, in link $l \in L$, that the network (operator) charges. By Lemma 4.1, the price for a service of type $s$ is $\bar{w}_{s}=\left\langle\overline{\boldsymbol{\lambda}}^{1}, \overline{\boldsymbol{\mu}}_{s}\right\rangle+\left\langle\overline{\boldsymbol{\lambda}}^{2}, \overline{\boldsymbol{\beta}}_{s}\right\rangle$ and the corresponding offered amount is $\bar{x}_{s}=\lambda_{s} \widehat{T} \exp \left(-\bar{w}_{s}\right)$.

The proof of part (ii) of the theorem is now complete.

In the above stated game, if player $N$ (the network operator) chooses the prices $\left(\overline{\boldsymbol{\lambda}}^{1}, \bar{\lambda}^{2}\right)$ (defined in the proof of Lemma 4.2) then, because of Lemma 4.2, each player $U_{s}$ (the user of service $s$ ) will request $\left(\bar{\mu}_{i}^{s}, \bar{\beta}_{i}^{s}\right)_{i \in r_{s}}$ units of bandwidth and buffers along the route $r_{s}, s \in S$. This welfare maximizing allocation is the result of the following tâtonnement [1]. At stage $n, n=0,1,2, \ldots$, the network operator announces a rent $\left(\lambda_{n, l}^{1}, \lambda_{n, l}^{2}\right)_{l \in L}$ for each unit of resources (bandwidth and buffers) at each link. Based on these prices, each user $U_{s}$ requests a certain amount of resources $\left(\mu_{i, n+1}^{s}, \beta_{i, n+1}^{s}\right)_{i \in r_{s}}$ for each type of service $s \in S$. These requests are

$$
\left(\mu_{i, n+1}^{s}, \beta_{i, n+1}^{s}\right)_{i \in r_{s}} \triangleq\left(\boldsymbol{\mu}_{s, n+1}, \boldsymbol{\beta}_{s, n+1}\right)=\arg \min _{\left(\boldsymbol{\mu}_{s}, \boldsymbol{\beta}_{s}\right) \in K_{s}}\left(\left\langle\boldsymbol{\lambda}_{n}^{1}, \boldsymbol{\mu}_{s}\right\rangle+\left\langle\boldsymbol{\lambda}_{n}^{2}, \boldsymbol{\beta}_{s}\right\rangle\right) .
$$

Upon receiving all the requests $\left(\boldsymbol{\mu}_{n+1}, \boldsymbol{\beta}_{n+1}\right)$ the network readjusts its prices to

$$
\left(\lambda_{l, n+1}^{1}, \lambda_{l, n+1}^{2}\right)_{l \in L} \triangleq\left(\boldsymbol{\lambda}_{n+1}^{1}, \boldsymbol{\lambda}_{n+1}^{2}\right)=\arg \min _{\left(\boldsymbol{\lambda}^{1}, \boldsymbol{\lambda}^{2}\right) \geqslant 0} G\left(\boldsymbol{\mu}_{n+1}, \boldsymbol{\beta}_{n+1}, \boldsymbol{\lambda}^{1}, \boldsymbol{\lambda}^{2}\right),
$$

and the process continues as described above.

Several comments are in order. 
(1) The tâtonnement leading to the welfare maximizing solution is informationally decentralized in nature. Given the prices $\left(\lambda^{1}, \lambda^{2}\right)$, the bandwidth and buffer requests that minimize the service cost per request and satisfy the quality of service requirements, depend only on the characteristics of the type of service requested (i.e., on the upper bound on the burstiness of the incoming traffic, the maximum tolerable percentage of loss and end-to-end delays) and on the (fixed) route designated for that service. All this information is available to the user requesting the service. The user's request is made without any knowledge of other users and service requests present in the network. On the other hand, the network operator has no knowledge of the number of users that are going to request service, or of the types of service requests. The network operator readjusts the prices per unit of bandwidth and buffer at each link based only on the users' response to the latest prices and on the knowledge of the aggregate demand for each type of service (cf. Assumption (A8)). Thus, the decentralized nature of the tatonnement described above is a striking feature of our solution. This feature is similar to the characteristics of the solution of the service provisioning problem formulated in [16].

(2) Contrary to the situation in [16] (where the determination of the resources required at each link of a fixed route is done locally at the corresponding node), nodes along a specific route for a specific service cannot make independent allocation decisions. This difference arises because the quality of service requirements included in our problem formulation, couple the decisions (allocations) made in different nodes along a route of a specific service. The coupling among allocations at individual links of a fixed route is a result of the interaction between resource allocation, percentage of cell loss and end-to-end cell delay.

(3) The optimal prices $\left(\bar{\lambda}^{1}, \bar{\lambda}^{2}\right)$, set by the network, determine a minimum cost allocation $(\overline{\boldsymbol{\mu}}, \overline{\boldsymbol{\beta}})$ for the users; $\left(\overline{\boldsymbol{\mu}}, \overline{\boldsymbol{\beta}}, \overline{\boldsymbol{\lambda}}^{1}, \overline{\boldsymbol{\lambda}}^{2}\right)$ determine, for each type of service $s$, the price $\bar{w}_{s}=\left\langle\overline{\boldsymbol{\lambda}}^{1}, \overline{\boldsymbol{\mu}}_{s}\right\rangle+\left\langle\overline{\boldsymbol{\lambda}}^{2}, \overline{\boldsymbol{\beta}}_{s}\right\rangle$ per request and the amount $\bar{x}_{s}=\lambda_{s} \widehat{T} \exp \left(-\bar{w}_{s}\right)$ of service $s \in S$, that is produced (cf. Lemma 4.1).

In the next Section we discuss the characteristics of the service provisioning procedure.

\section{Conclusions - reflections}

We have presented an approach to Service Provisioning with Quality of Service requirements in ATM networks.

The main contribution of the paper is the incorporation of quality of service requirements into the service provisioning approach originally proposed in [16]. Our approach to analyzing quality of service in ATM networks is intuitively appealing as it captures the interaction among resource allocation, percentage of cell loss, and end-to-end cell delays along the fixed routes that provide the connection for the different types of services.

The results of our analysis of quality of service can also be used to address the admission control problem in ATM networks as follows: When the network receives a request for the admission of a certain type of service $s(s \in S)$, it checks its available resources, and attempts to identify a route along which there is an allocation of available bandwidth and available buffers that satisfies the requirements on percentage of losses and delay. The requirements are expressed by Eqs (4.7) and (4.8), where the left-hand-sides of (4.7) and (4.8) are given by expressions similar to (3.42) and (3.49), respectively. If such an allocation exists, the network admits the service request, otherwise it rejects it.

The main features of our approach are:

(1) The network model is deterministic. A fluid approximation is used to describe the flow of messages that travel through the network. Input messages are categorized into different types of services according to their maximum input rate, the upper bound on their burstiness curve, and their Quality of Service requirements expressed by the maximum percentage of cell loss, maximum end-to-end cell delay, and maximum jitter.

(2) There is no sharing of resources (bandwidth and buffers) at each ATM switch among the different virtual circuits that pass through the switch. The implications of this feature of our approach are: (i) the behavior of one connection does not affect the quality of service of other connections that share the same nodes; and (ii) jitter is zero. 
(3) Service provisioning is achieved through an iterative pricing scheme, where at each step of the iteration the network sets prices for its resources and users respond by requesting a resource allocation along their entire route that satisfies their quality of service requirements (and minimizes the cost of their service) (cf. Section 4).

(4) To incorporate quality of service requirements into service provisioning we take a worst case approach. For a fixed resource allocation (bandwidth and buffers) along the route of a service we derive expressions for the maximum percentage of loss and the maximum end-to-end cell delay for that service. We require that the maximum percentage of loss and the maximum end-to-end cell delay remain below certain limits.

(5) Centralization of information is not required by our approach. The network needs to know only the average request rate of each type of service, the route of each service, the resources requested by the users, and the time each admitted service terminates. The users need to know only their input traffic constraints, their quality of service requirements, and the prices the network announces for its resources.

(6) Contrary to the situation in [16] the determination of resources required at each link of a fixed service route has to be made jointly by all the nodes along the route. This coupling among the nodes of a fixed route, corresponding to a certain type of service, arises because of the quality of service requirements (for that service) that are included in the formulation of the service provisioning problem.

(7) The network reallocates its available resources every $\widehat{T}$ seconds. At allocation instances resources are not reserved in anticipation of future requests. The determination of an optimal or near optimal value of $\widehat{T}$ remains an open and challenging problem. Its selection must take into account the overall delay constraints (end-to-end cell delay plus the delay due to waiting for the next allocation epoch) of the different types of services, the arrival rates of incoming traffic, and the duration of the different types of services.

Important open problems, some of which will be addressed in future work, are: (i) the investigation of resource sharing among services and service scheduling at each ATM switch (cf. discussion of Section 2); (ii) the incorporation of routing as an optimization component into the overall service provisioning approach; and (iii) the comparison of the approach developed here with service provisioning approaches using stochastic network models.

\section{Acknowledgements}

This research was supported in part by NSF Grant NCR-9204419 and ARO Grant DAAH04-96-1-0377. We would like to thank Professor Gustavo de Veciana for bringing to our attention a mistake that we had on a previous version of our paper. We are grateful to the anonymous reviewers whose excellent comments significantly improved this paper. Finally we would like to thank Professor Steve Chick and Mr Socrates Vamvakos for useful discussions.

\section{References}

[1] K.J. Arrow and F.H. Hahn, General Competitive Analysis, Holden-Day, San Francisco, 1971

[2] R.L. Cruz, Quality of service guarantees in virtual circuit switched networks, IEEE J. Select. Areas Commun. 13 (1995), $1048-1056$.

[3] M. Decina, T. Toniatti, P. Vaccari and L. Verri, Bandwidth assignment and virtual call blocking in ATM networks, in: Proc. IEEE INFOCOM, 1990, pp. 881-888.

[4] A. Demers, S. Keshav and S. Shenker, Analysis and simulation of a fair queueing algorithm, in: Proc. ACM SIGCOMM, 1989, pp. $3-12$.

[5] D. Ferrari and D. Verma, A scheme for real-time channel establishment in wide area networks, IEEE J. Select. Areas Commun. 8 (1990), 368-379.

[6] G. Gallassi, G. Rigolio and L. Fratta, ATM: bandwidth assignment and bandwidth enforcement policies, in: Proc. GLOBECOM, 1989, pp. 1788-1793. 
[7] L. Georgiadis, R. Guerin, V. Peris and K.N. Sivarajan, Efficient network QoS provisioning based on per node traffic shaping, IEEE/ACM Trans. Networking 4 (1996), 482-501.

[8] R.J. Gibbens and P.J. Hunt, Effective bandwidths for the multi-type UAS channel, Queueing Systems 9(1-2) (1991), 17-27.

[9] S.J. Golestani, Congestion-free communication in high-speed packet networks, IEEE Trans. Commun. 39 (1991), 1802-1812.

[10] R. Guerin, H. Ahmadi and M. Naghshineh, Equivalent capacity and its application to bandwidth allocation in high-speed networks, IEEE J. Select. Areas Commun. 9 (1991), 968-981.

[11] C. Kalmanek, H. Kanakia and S. Keshav, Rate controlled servers for very high speed networks, in: Proc. GLOBECOM, 1990, 12-20.

[12] F.P. Kelly, Effective bandwidths at multi-class queues, Queueing Systems 9(1-2) (1991), 5-15.

[13] G. Kesidis, J. Walrand and C.S. Chang, Effective bandwidths for multiclass Markov fluids and other ATM sources, IEEE/ACM Trans. Networking 1 (1993), 424-428.

[14] E.W. Knightly, H-BIND: A new approach to providing statistical performance guarantees to VBR traffic, in: Proc. IEEE INFOCOM, 1996, pp. 1091-1099.

[15] S. Low and P. Varaiya, A simple theory of traffic and resource allocation in ATM, in: Proc. GLOBECOM, 1991, pp. $1633-1637$.

[16] S. Low and P. Varaiya, A new approach to service provisioning in ATM networks, IEEE/ACM Trans. Networking 1 (1993), $547-553$. See also [17] for corrections.

[17] S. Low and P. Varaiya, Corrections to: A new approach to service provisioning in ATM Networks, IEEE/ACM Trans. Networking 2 (1994), 312.

[18] S. Low and P. Varaiya, Burst reducing servers in ATM networks, Queueing Systems 20 (1995), 61-84.

[19] A. Mas-Colell, M.D. Whinston and J.R. Green, Microeconomic Theory, Oxford University Press, New York, 1995.

[20] D. Mitra, J.A. Morrison and K.G. Ramakrishnam, ATM network design and optimization: A multirate loss network framework, IEEE/ACM Trans. Networking 4 (1996), 531-543.

[21] A.K. Parekh and R.G. Gallager, A generalized processor sharing approach to flow control in integrated services networks: The single-node case, IEEE/ACM Trans. Networking 1 (1993), 344-357.

[22] A.K. Parekh and R.G. Gallager, A generalized processor sharing approach to flow control in integrated services networks: The multiple-node case, IEEE/ACM Trans. Networking 2 (1994), 137-150.

[23] P.A. Samuelson and W.D. Nordhaus, Economics, McGraw-Hill, New York, 1992.

[24] P. Thomas and D. Teneketzis, An approach to service provisioning with quality of service requirements in ATM networks, Control Group Report CGR 96-11, Department of Electrical Engineering and Computer Science, University of Michigan, 1996.

[25] D.C. Verma, H. Zhang and D. Ferrari, Guaranteeing delay jitter bounds in packet switching networks, in: Proc. TRICOMM, Chapel Hill, North Carolina, 1991, pp. 35-43.

[26] M. Wong and P. Varaiya, A deterministic fluid model for cell loss in ATM networks, in: Proc. IEEE INFOCOM, 1993, pp. 395-400.

[27] L. Zhang, Virtual clock: A new traffic control algorithm for packet-switched networks, ACM Trans. Computer Systems 9 (1991), $101-124$. 